\title{
CHARACTERIZATION OF TANK 11H AND TANK 51H POST ALUMINUM DISSOLUTION PROCESS SAMPLES
}

May 2008

Waste Processing Technology Section Savannah River National Laboratory Aiken, SC 29808

Prepared for the U.S. Department of Energy Under Contract Number DEAC09-96SR18500

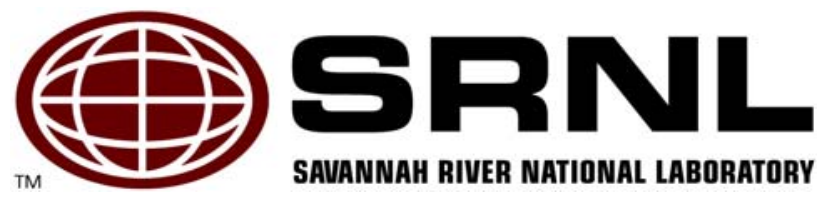




\title{
DISCLAIMER
}

This report was prepared for the United States Department of Energy under Contract No. DE-AC09-96SR18500 and is an account of work performed under that contract. Neither the United States Department of Energy, nor WSRC, nor any of their employees makes any warranty, expressed or implied, or assumes any legal liability or responsibility for the accuracy, completeness, or usefulness, of any information, apparatus, or product or process disclosed herein or represents that its use will not infringe privately owned rights. Reference herein to any specific commercial product, process, or service by trade name, trademark, name, manufacturer or otherwise does not necessarily constitute or imply endorsement, recommendation, or favoring of same by Westinghouse Savannah River Company or by the United States Government or any agency thereof. The views and opinions of the authors expressed herein do not necessarily state or reflect those of the United States Government or any agency thereof.

\author{
Printed in the United States of America \\ Prepared For \\ U.S. Department of Energy
}


Key Words:

Sludge Analysis

Supernate Analysis

\title{
Retention: Permanent
}

\section{Key References:}

Technical Task Request: PIT-TTR-2007-00006, Rev. 0

Technical Task Plan: WSRC-RP-2007-01313, Rev. 0

\section{CHARACTERIZATION OF TANK 11H AND TANK 51H POST ALUMINUM DISSOLUTION PROCESS SAMPLES}

\author{
M. S. Hay, SRNL/A\&CPT \\ D. J. McCabe, SRNL/A\&CPT
}

Issue Date: May 2008

Waste Processing Technology Section Savannah River National Laboratory Aiken, SC 29808

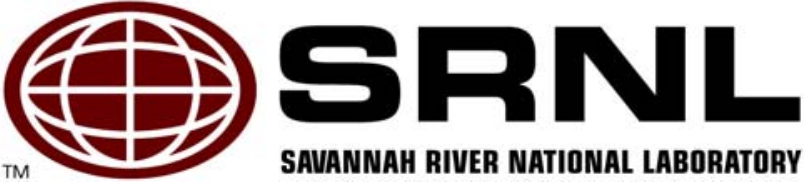


WSRC-STI-2008-00227, REV. 0

\section{REVIEWS AND APPROVALS}

M. S. Hay, Author, SRNL/A\&CPT

Date

D. J. McCabe, Author, SRNL/A\&CPT

Date

S. H. Reboul, Technical Reviewer, SRNL/PS\&E

Date

J. Stuberfield, Project Owner, Technology Development and Tank Closure

Date 


\section{TABLE OF CONTENTS}

List of Tables $\quad$ iv

$\begin{array}{ll}\text { List of Acronyms } & \text { v }\end{array}$

SUMMARY vi vi v

$\begin{array}{ll}1.0 \text { INTRODUCTION } & 1\end{array}$

2.0 SAMPLE CHARACTERIZATION 2

2.1 SAMPLE DESCRIPTION $\quad 2$

2.2 SUPERNATE SAMPLE PREPARATION FOR ANALYSIS $\quad 2$

2.3 SLUDGE SAMPLE PREPARATION FOR ANALYSIS 2

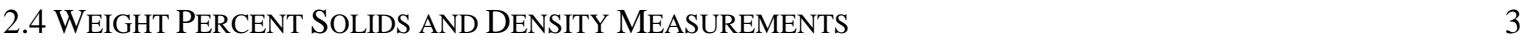

3.0 RESULTS OF THE SAMPLE CHARACTERIZATION

3.1 GENERAL INFORMATION

5

3.2 Weight PERCENT SOlids AND DENSITY RESUlts OF THE AS-RECEIVED SLUDGE SLURRY SAMPLE

3.3 ANALYTICAL RESULTS FOR THE TANK 11H AND TANK 51H SUPERNATE

3.4 ANALYTICAL RESULTS FOR THE AS-RECEIVED TANK 51H SLUDGE SOLIDS

\subsection{DISCUSSION OF RESULTS 24}

4.1 As-RECEIVED TANK 11H AND TANK 51H SUPERNATE CHARACTERIZATION

\subsection{REFERENCES}




\section{List of Tables}

TABLE 2-1. COMPOSITION OF THE ANALYTICAL REFERENCE GLASS-1 STANDARD..........................................

TABLE 3-1. ABBREVIATIONS FOR ANALYTICAL METHODS USED IN DATA TABLES ............................................5

TABle 3-2. WEIGHT PERCENT SOLIDS AND DENSITY OF THE AS-RECEIVED TANK 11H Dip SAMPLE ..................7

Table 3-3. Weight Percent Solids And Density of The As-Received TANK 51H Sludge SLuRry SAMPLE .7

TABLE 3-4. COMPOSITION OF THE AS-RECEIVED TANK 11H SUPERNATE ....................................................

TABLE 3-5. COMPOSITION OF THE AS-RECEIVED TANK 51H FILTERED SUPERNATE .......................................12

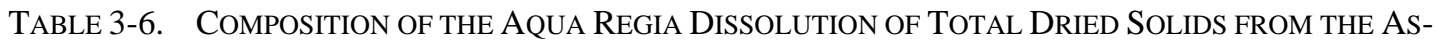
RECEIVED TANK 51H SLUDGE......................................................................................... 16

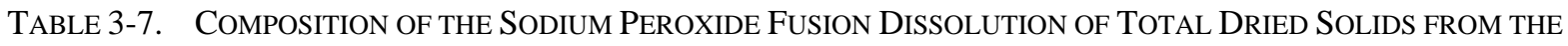
AS-RECEIVED TANK 51H SLUDGE .......................................................................................20

TABLE 4-1. COMPARISON OF THE TANK 11H AND TANK 51H SUPERNATE..................................................24

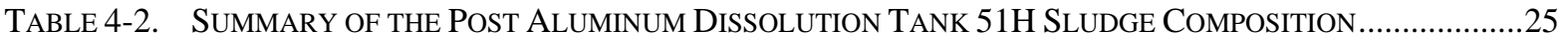




\section{List of Acronyms}

$\begin{array}{ll}\text { SRS } & \text { Savannah River Site } \\ \text { LWO } & \text { Liquid Waste Organization } \\ \text { HM } & \text { H-Area Modified PUREX } \\ \text { LTAD } & \text { Low Temperature Aluminum Dissolution } \\ \text { DWPF } & \text { Defense Waste Processing Facility } \\ \text { SRNL } & \text { Savannah River National Laboratory } \\ \text { ARG-1 } & \text { Analytical Reference Glass 1 } \\ \text { AD } & \text { Analytical Development } \\ \text { \%RSD } & \text { Percent Relative Standard Deviation } \\ \text { IE } & \text { Inductively Couple Plasma-Emission Spectroscopy } \\ \text { IM } & \text { Inductively Couple Plasma-Mass Spectrometry } \\ \text { IC } & \text { Ion Chromatography } \\ \text { TH } & \text { Titration for Free Hydroxide } \\ \text { TIC } & \text { Total Inorganic Carbon } \\ \text { CV } & \text { Atomic Absorption Cold Vapor Method } \\ \text { SG } & \text { Separation/Gamma Spectroscopy } \\ \text { SA } & \text { Separation/Alpha Spectroscopy } \\ \text { SL } & \text { Separation/Liquid Scintillation } \\ \text { HM } & \text { H-Area Modified PUREX }\end{array}$




\section{SUMMARY}

A dip sample of the liquid phase from Tank $11 \mathrm{H}$ and a 3-L slurry sample from Tank $51 \mathrm{H}$ were obtained and sent to Savannah River National Laboratory for characterization. These samples provide data to verify the amount of aluminum dissolved from the sludge as a result of the low temperature aluminum dissolution process conducted in Tank $51 \mathrm{H}$.

The characterization results for the as-received Tank $11 \mathrm{H}$ and Tank $51 \mathrm{H}$ supernate samples and the total dried solids of the Tank 51H sludge slurry sample appear quite good with respect to the precision of the sample replicates and minimal contamination present in the blank. The two supernate samples show similar concentrations for the major components as expected. 


\subsection{INTRODUCTION}

The Savannah River Site (SRS) Liquid Waste Organization (LWO) added caustic to the HArea Modified PUREX (HM) sludge in Tank 51H to dissolve aluminum using a low temperature aluminum dissolution (LTAD) process. The LTAD process was conducted to reduce the total mass of sludge solids being fed to the Defense Waste Processing Facility (DWPF). After completion of the LTAD, the sludge was allowed to settle for approximately one month. Once settling was complete the aluminum rich supernate was decanted from Tank $51 \mathrm{H}$ to Tank $11 \mathrm{H}$. Additional caustic was added to the small amount of sludge and supernate in Tank $11 \mathrm{H}$ prior to the transfer of the aluminum rich supernate from Tank $51 \mathrm{H}$. A dip sample of the liquid phase from Tank $11 \mathrm{H}$ and a 3-L slurry sample from Tank $51 \mathrm{H}$ were obtained and sent to Savannah River National Laboratory (SRNL) for characterization. A Technical Task Request ${ }^{1}$ detailing the required characterization and a Task Technical and Quality Assurance Plan² were generated for the task. 
WSRC-STI-2008-00227, REV. 0

\subsection{SAMPLE CHARACTERIZATION}

\subsection{SAMPLE DESCRIPTION}

A 3-liter sludge slurry sample from Tank 51H (ID No. = HTF-51-07-121) and a $200 \mathrm{~mL}$ dip sample from Tank 11H (ID No. = HTF-11-07-122) were sent to SRNL for characterization. The samples were received at SRNL on February 15, 2008. The sludge slurry sample was mixed and pumped from the sample container. The sludge sample was allowed to settle overnight after which clear supernate was pumped back into the sample container to rinse out any remaining sludge solids. Approximately $3.05 \mathrm{~L}$ of a sludge solids/supernate mixture was recovered from the sample container. The Tank $11 \mathrm{H}$ dip sample was vigorously shaken to mix the sample and transferred to a poly bottle. The weight of sample recovered from the dip bottle was $165.135 \mathrm{~g}(\sim 140 \mathrm{~mL})$ and with no visible solids in the sample.

\subsection{SUPERNATE SAMPLE PREPARATION FOR ANALYSIS}

Portions of the Tank $11 \mathrm{H}$ dip sample were prepared for analysis by dilution in de-ionized distilled water or nitric acid to reduce the sample activity and allow removal from the Shielded Cells. The sample was not filtered prior to sample preparation at the request of the customer. All sample preparations of the unfiltered Tank $11 \mathrm{H}$ supernate were conducted in triplicate. A blank was prepared along with the sample dilutions.

A portion of the well-mixed Tank $51 \mathrm{H}$ sludge slurry was filtered through a $0.45 \mu$ porosity filter. Portions of the filtered supernate were diluted with de-ionized distilled water or nitric acid to reduce the sample activity and allow removal from the Shielded Cells for chemical analysis. All sample preparations of the filtered Tank $51 \mathrm{H}$ supernate were conducted in triplicate. A blank was prepared along with the sample dilutions.

\subsection{SLUDGE SAMPLE PREPARATION FOR ANALYSIS}

Portions of the Tank $51 \mathrm{H}$ sludge slurry were dried in an oven at $100{ }^{\circ} \mathrm{C}$. The dried solids were prepared for analysis using two methods; dissolution in aqua regia, and fusion with sodium peroxide followed by uptake in nitric acid. The digested solids were diluted to reduce activity and allow removal from the Shielded Cells. All sample preparations were conducted in triplicate. Duplicate digestions of a glass standard containing many of the elements found in tank waste sludge samples were prepared concurrently with the sample digestions to check for the completeness of the dissolution and contamination levels introduced during sample preparation. Table 2-1 lists the composition of the Analytical Reference Glass-1 (ARG-1) glass standard. ${ }^{3}$ A reagent blank was prepared along with the sample digestions.

For the ${ }^{79}$ Se and ${ }^{129}$ I analysis, special sample preparation instructions were supplied by Analytical Development section (AD) using aliquots of the undried slurry sample. 
Table 2-1. Composition of the Analytical Reference Glass-1 Standard.

\begin{tabular}{|l|c|c|}
\hline Element & wt \% in Glass & $\mathbf{~ m g / k g ~ i n ~ G l a s s ~}$ \\
\hline $\mathrm{Al}$ & 2.50 & $2.50 \mathrm{E}+04$ \\
\hline $\mathrm{B}$ & 2.69 & $2.69 \mathrm{E}+04$ \\
\hline $\mathrm{Ba}$ & 0.079 & $7.90 \mathrm{E}+02$ \\
\hline $\mathrm{Ca}$ & 1.02 & $1.02 \mathrm{E}+04$ \\
\hline $\mathrm{Cr}$ & 0.064 & $6.40 \mathrm{E}+02$ \\
\hline $\mathrm{Cu}$ & 0.003 & $3.0 \mathrm{E}+01$ \\
\hline $\mathrm{Fe}$ & 9.79 & $9.79 \mathrm{E}+04$ \\
\hline $\mathrm{K}$ & 2.26 & $2.26 \mathrm{E}+04$ \\
\hline $\mathrm{Li}$ & 1.49 & $1.49 \mathrm{E}+04$ \\
\hline $\mathrm{Mg}$ & 0.52 & $5.2 \mathrm{E}+03$ \\
\hline $\mathrm{Mn}$ & 1.46 & $1.46 \mathrm{E}+04$ \\
\hline $\mathrm{Na}$ & 8.52 & $8.52 \mathrm{E}+04$ \\
\hline $\mathrm{Ni}$ & 0.83 & $8.3 \mathrm{E}+03$ \\
\hline $\mathrm{P}$ & 0.11 & $1.1 \mathrm{E}+03$ \\
\hline $\mathrm{Si}$ & 22.4 & $2.24 \mathrm{E}+05$ \\
\hline $\mathrm{Sr}$ & 0.003 & $3.0 \mathrm{E}+01$ \\
\hline $\mathrm{Ti}$ & 0.69 & $6.9 \mathrm{E}+03$ \\
\hline $\mathrm{Zn}$ & 0.016 & $1.6 \mathrm{E}+02$ \\
\hline $\mathrm{Zr}$ & 0.096 & $9.6 \mathrm{E}+02$ \\
\hline
\end{tabular}

\subsection{WEIGHT PERCENT SOLIDS AND DENSITY MEASUREMENTS}

The densities of the filtered supernate and the well-mixed slurry sample were measured in the Shielded Cells using calibrated plastic tubes with a nominal volume of $\sim 8.25 \mathrm{~mL}$. The density measurements were conducted in triplicate on each sample.

The weight percent total solids in the slurry sample and the weight percent dissolved solids in the supernate were measured in the Shielded Cells using a conventional drying oven at 105 ${ }^{\circ} \mathrm{C}$. The sample was dried until repeated weights indicated no further loss of water. The weight percent dissolved solids in a sample of the filtered supernate were measured in the same manner. All weight percent solids measurements were made in triplicate. The weight percent insoluble solids and weight percent soluble solids in the slurry sample were calculated using the equations shown below. 
Wis $=($ Wts - Wds $) /(1-W d s) \quad$ and $\quad$ Wss $=$ Wts - Wis

where:

Wis = weight fraction of insoluble solids in the slurry

Wts = weight fraction of total solids in the slurry

Wds = weight fraction of dissolved solids in the filtered supernate

Wss = weight fraction of soluble solids in the slurry

Thus:

$\mathrm{Wt} \%$ dissolved solids $=$ $\mathrm{Wt} \%$ total solids =

$\mathrm{Wt} \%$ insoluble solids = $\mathrm{Wt} \%$ soluble solids $=$ (wt dissolved solids/wt of supernate) x 100

(wt total solids/wt of total slurry) $\mathrm{x} 100$

(wt insoluble solids/wt of total slurry) x 100

(wt of dissolved solids/wt of total slurry) x 100 


\subsection{RESULTS OF THE SAMPLE CHARACTERIZATION}

\subsection{GENERAL INFORMATION}

The data tables at the end of the section provide the composition of the Tank $11 \mathrm{H}$ and Tank $51 \mathrm{H}$ samples received at SRNL. Portions of these results were reported previously. ${ }^{4}$

The tables include the results of all replicates, blanks, and glass standards. In tables containing data for digested solids samples, the blank was converted to a dried solids basis to allow direct comparison to the sample data as a quality indicator. The data for the blank were converted to a dried solids basis by dividing the concentrations measured in the blank by the target weight of solids used in the sample digestions ( $\sim 0.250 \mathrm{~g}$ in most cases). For waste tank sample characterization, an uncertainty of approximately $+/-15 \%$ has been found to be the normal range for the combined sampling and analytical uncertainty. ${ }^{5}$ For the Tank $11 \mathrm{H}$ and Tank $51 \mathrm{H}$ samples, the percent relative standard deviation (\%RSD) presented in the tables only includes the uncertainty associated with sub-sampling in the Shielded Cells and the uncertainty of the analytical method. It should be noted that the samples represent a small amount of material from a large tank.

The data tables also indicate the analytical method used to measure each analyte. Table 3-1 shows the abbreviations used for each analytical method:

Table 3-1. Abbreviations for Analytical Methods used in Data Tables

\begin{tabular}{|l|c|}
\hline Analytical Method & $\begin{array}{c}\text { Abbreviation } \\
\text { in Tables }\end{array}$ \\
\hline Inductively Coupled Plasma-Emission Spectroscopy & IE \\
\hline Inductively Coupled Plasma-Mass Spectrometry & IM \\
\hline Ion Chromatography & IC \\
\hline Titration for Free Hydroxide & TH \\
\hline Total Inorganic Carbon & TIC \\
\hline Atomic Absorption Cold Vapor Method & CV \\
\hline Separation/Gamma Spec. & SG \\
\hline Separation/Alpha Spec. & SA \\
\hline Separation/Liquid Scintillation & SL \\
\hline
\end{tabular}


WSRC-STI-2008-00227, REV. 0

\subsection{WEIGHT PERCENT SOLIDS AND DENSITY RESULTS OF THE AS- RECEIVED SLUDGE SLURRY SAMPLE}

Tables 3-2 and 3-3 show the results of weight percent solids and density measurements made on the as-received Tank $11 \mathrm{H}$ sample, the as-received Tank $51 \mathrm{H}$ sludge slurry sample and the filtered supernate obtained from the Tank $51 \mathrm{H}$ sludge slurry sample.

\subsection{ANALYTICAL RESULTS FOR THE TANK 11H AND TANK 51H SUPERNATE}

Table 3-4 shows the results of the analysis of the as-received Tank $11 \mathrm{H}$ (unfiltered) supernate. The anion/cation charge balance for the supernate appears good with a difference of only $2 \%$. The sulfate concentration measured by IC shows good agreement with the sulfur concentration measured by IE (6\% difference). The generally low percent relative standard deviations for all of the analyses on the supernate sample indicate good precision between the three sample replicates. The blank prepared with the sample replicates shows no measurable contamination.

Table 3-5 shows the results of the analysis of the as-received Tank $51 \mathrm{H}$ filtered supernate. Again, the anion/cation charge balance for the supernate appears quite good with a difference of only $8 \%$. The sulfate concentration measured by IC shows good agreement with the sulfur concentration measured by IE (3\% difference). The low percent relative standard deviations for all of the analyses on the supernate sample show good precision between the three sample replicates. However, in the IC analysis for nitrate, one of the sample replicates shows a result $>50 \%$ higher than the average of the other two sample replicates. This value was dropped from the calculations of the average and percent relative standard deviation. The blank prepared with the sample replicates shows slight contamination with ${ }^{137} \mathrm{Cs}$ and ${ }^{99} \mathrm{Tc}$. The low concentration of the ${ }^{137} \mathrm{Cs}$ contamination in the blank relative to the measured concentration of ${ }^{137} \mathrm{Cs}$ in the samples indicates the cesium contamination should not impact the sample analysis results. However, the ${ }^{99}$ Tc contamination in the blank shows a concentration only $\sim 3 \mathrm{X}$ lower than the ${ }^{99}$ Tc concentration measured in the samples. The ${ }^{99}$ Tc contamination could bias the sample results high.

\subsection{ANALYTICAL RESULTS FOR THE AS-RECEIVED TANK 51H SLUDGE SOLIDS}

Tables 3-6 and 3-7 show the results of the analysis of the aqua regia and sodium peroxide fusion dissolutions of the total dried solids obtained from the as-received Tank $51 \mathrm{H}$ sludge slurry sample (HTF-51-07-121). The aqua regia and sodium peroxide fusion data show good agreement between the two dissolution methods for the major components of the sludge solids. The ${ }^{239 / 240} \mathrm{Pu}$ results by IM and alpha counting show poor agreement. The IM results indicate lower concentrations of plutonium than the counting results. The ${ }^{239 / 240} \mathrm{Pu}$ show good agreement with the values measured in the post aluminum dissolution sludge from the 3-L aluminum dissolution demonstration. ${ }^{6}$ Prior experience seems to indicate that the aqua regia dissolution method will not consistently dissolve all of the aluminum in the current Tank $51 \mathrm{H}$ sludge batch. ${ }^{6}$ However, the aqua regia results for aluminum on the current sample show good agreement with sodium peroxide fusion results. 
WSRC-STI-2008-00227, REV. 0

Table 3-2. Weight Percent Solids and Density of the As-Received Tank 11H Dip Sample

\begin{tabular}{|l|c|c|c|c|c|c|}
\hline Analyte (Method) & Units & $\begin{array}{c}\mathbf{1}^{\text {st }} \\
\text { Replicate }\end{array}$ & $\begin{array}{c}\mathbf{2}^{\text {nd }} \\
\text { Replicate }\end{array}$ & $\begin{array}{c}3^{\text {rd }} \\
\text { Replicate }\end{array}$ & Average & \%RSD \\
\hline Weight Percent Dissolved Solids & $\mathrm{Wt} \%$ & $18.5 \%$ & $18.3 \%$ & $18.4 \%$ & $18.4 \%$ & $0.4 \%$ \\
\hline Density of Supernate & $\mathrm{g} / \mathrm{mL}$ & 1.18 & 1.17 & 1.17 & 1.17 & $0.5 \%$ \\
\hline
\end{tabular}

Table 3-3. Weight Percent Solids and Density of the As-Received Tank 51H Sludge Slurry Sample

\begin{tabular}{|c|c|c|c|c|c|c|}
\hline Analyte (Method) & Units & $\begin{array}{c}1^{\text {st }} \\
\text { Replicate } \\
\end{array}$ & $\begin{array}{c}2^{\text {nd }} \\
\text { Replicate }\end{array}$ & $\begin{array}{c}3^{\text {rd }} \\
\text { Replicate } \\
\end{array}$ & Average & \%RSD \\
\hline Weight Percent Total Solids & $\mathrm{Wt} \%$ & $23.4 \%$ & $23.8 \%$ & $23.7 \%$ & $23.6 \%$ & $0.9 \%$ \\
\hline Weight Percent Dissolved Solids & $\mathrm{Wt} \%$ & $19.4 \%$ & $19.4 \%$ & $19.0 \%$ & $19.3 \%$ & $1.2 \%$ \\
\hline Weight Percent Soluble Solids & $\mathrm{Wt} \%$ & - & - & - & $18.2 \% *$ & - \\
\hline Weight Percent Insoluble Solids & $\mathrm{Wt} \%$ & - & - & - & $5.41 \% *$ & - \\
\hline Density of Slurry & $\mathrm{g} / \mathrm{mL}$ & 1.21 & 1.23 & 1.23 & 1.22 & $0.9 \%$ \\
\hline Density of Supernate & $\mathrm{g} / \mathrm{mL}$ & 1.20 & 1.20 & 1.21 & 1.20 & $0.5 \%$ \\
\hline
\end{tabular}

* Values for the weight percent soluble solids and weight percent insoluble solids were calculated from the measured weight percent total solids and weight percent dissolved solids (see Section 2.4) 
WSRC-STI-2008-00227, REV. 0

Table 3-4. Composition of the As-Received Tank 11H Supernate

\begin{tabular}{|c|c|c|c|c|c|c|c|}
\hline Analyte (Method) & Units & $\begin{array}{c}1^{\text {st }} \\
\text { Replicate } \\
\end{array}$ & $\begin{array}{c}2^{\text {nd }} \\
\text { Replicate } \\
\end{array}$ & $\begin{array}{c}3^{\text {rd }} \\
\text { Replicate } \\
\end{array}$ & Average & \%RSD & Blank \\
\hline $\mathrm{NO}_{3}^{-}$(IC) & M & $1.74 \mathrm{E}-01$ & $1.76 \mathrm{E}-01$ & $1.76 \mathrm{E}-01$ & $1.75 \mathrm{E}-01$ & $0.5 \%$ & - \\
\hline $\mathrm{NO}_{2}^{-}$(IC) & M & 3.26E-01 & 3.33E-01 & 3.30E-01 & 3.30E-01 & $1.0 \%$ & - \\
\hline $\mathrm{PO}_{4}{ }^{3-}(\mathrm{IC})$ & $\mathrm{M}$ & $<1.3 \mathrm{E}-02$ & $<1.3 \mathrm{E}-02$ & $<1.3 \mathrm{E}-02$ & - & - & - \\
\hline $\mathrm{SO}_{4}{ }^{2-}(\mathrm{IC})$ & $\mathrm{M}$ & $1.76 \mathrm{E}-02$ & $1.74 \mathrm{E}-02$ & 1.73E-02 & $1.74 \mathrm{E}-02$ & $0.8 \%$ & - \\
\hline $\mathrm{C}_{2} \mathrm{O}_{4}{ }^{2-}$ (IC) & $\mathrm{M}$ & $<5.7 \mathrm{E}-03$ & $<5.7 \mathrm{E}-03$ & $<5.7 \mathrm{E}-03$ & - & - & - \\
\hline $\mathrm{Cl}^{-}(\mathrm{IC})$ & M & 3.10E-02 & 2.91E-02 & 2.91E-02 & 2.97E-02 & $3.8 \%$ & - \\
\hline $\mathrm{F}^{-}$(IC) & M & $<2.6 \mathrm{E}-02$ & $<2.6 \mathrm{E}-02$ & $<2.6 \mathrm{E}-02$ & - & - & - \\
\hline $\mathrm{CHO}_{2}^{-}$(IC) & M & $<1.1 \mathrm{E}-02$ & $<1.1 \mathrm{E}-02$ & $<1.1 \mathrm{E}-02$ & - & - & - \\
\hline $\mathrm{OH}_{\text {free }}^{-}(\mathrm{TH})$ & $\mathrm{M}$ & $3.13 E+00$ & $3.10 \mathrm{E}+00$ & $3.06 \mathrm{E}+00$ & $3.10 \mathrm{E}+00$ & $1.1 \%$ & - \\
\hline $\mathrm{CO}_{3}{ }^{2-}$ (TIC) & $\mathrm{M}$ & 9.92E-02 & $9.83 \mathrm{E}-02$ & $9.67 \mathrm{E}-02$ & 9.81E-02 & $1.3 \%$ & - \\
\hline Ag (IE) & $\mathrm{mg} / \mathrm{L}$ & $<5.7 \mathrm{E}+00$ & $<5.7 \mathrm{E}+00$ & $<5.7 \mathrm{E}+00$ & - & - & $<5.8 \mathrm{E}+00$ \\
\hline $\mathrm{Al}$ (IE) & $\mathrm{mg} / \mathrm{L}$ & $1.06 \mathrm{E}+04$ & $1.07 \mathrm{E}+04$ & $1.06 \mathrm{E}+04$ & $1.06 \mathrm{E}+04$ & $0.2 \%$ & $<2.6 \mathrm{E}+01$ \\
\hline B (IE) & $\mathrm{mg} / \mathrm{L}$ & $<4.9 \mathrm{E}+00$ & $<5.0 \mathrm{E}+00$ & $<4.9 \mathrm{E}+00$ & - & - & $<5.0 \mathrm{E}+00$ \\
\hline $\mathrm{Ba}$ (IE) & $\mathrm{mg} / \mathrm{L}$ & $<1.2 \mathrm{E}+00$ & $<1.2 \mathrm{E}+00$ & $<1.2 \mathrm{E}+00$ & - & - & $<1.2 \mathrm{E}+00$ \\
\hline Be (IE) & $\mathrm{mg} / \mathrm{L}$ & $<2.2 \mathrm{E}-01$ & $<2.2 \mathrm{E}-01$ & $<2.2 \mathrm{E}-01$ & - & - & $<2.2 \mathrm{E}-01$ \\
\hline $\mathrm{Ca}$ (IE) & $\mathrm{mg} / \mathrm{L}$ & $<1.6 \mathrm{E}+01$ & $<1.6 \mathrm{E}+01$ & $<1.6 \mathrm{E}+01$ & - & - & $<1.6 \mathrm{E}+01$ \\
\hline Cd (IE) & $\mathrm{mg} / \mathrm{L}$ & $2.76 \mathrm{E}+00$ & $1.86 \mathrm{E}+00$ & $1.83 \mathrm{E}+00$ & $2.15 \mathrm{E}+00$ & $24 \%$ & $<1.6 \mathrm{E}+00$ \\
\hline Ce (IE) & $\mathrm{mg} / \mathrm{L}$ & $<6.5 \mathrm{E}+01$ & $<6.5 \mathrm{E}+01$ & $<6.5 \mathrm{E}+01$ & - & - & $<6.5 \mathrm{E}+01$ \\
\hline Cr (IE) & $\mathrm{mg} / \mathrm{L}$ & $2.76 \mathrm{E}+01$ & $2.78 \mathrm{E}+01$ & $2.79 \mathrm{E}+01$ & $2.77 \mathrm{E}+01$ & $0.6 \%$ & $<2.0 \mathrm{E}+00$ \\
\hline $\mathrm{Cu}$ (IE) & $\mathrm{mg} / \mathrm{L}$ & $<3.2 \mathrm{E}+00$ & $<3.3 \mathrm{E}+00$ & $<3.3 \mathrm{E}+00$ & - & - & $<3.3 \mathrm{E}+00$ \\
\hline Fe (IE) & $\mathrm{mg} / \mathrm{L}$ & $9.81 E+00$ & $9.98 \mathrm{E}+00$ & $9.82 E+00$ & $9.87 \mathrm{E}+00$ & $0.9 \%$ & $<3.2 \mathrm{E}+00$ \\
\hline
\end{tabular}

The As-Received Tank 11 Supernate was not filtered prior to submitting samples for analysis at the request of the customer. 
WSRC-STI-2008-00227, REV. 0

Table 3-4. Composition of the As-Received Tank 11H Supernate (Continued)

\begin{tabular}{|c|c|c|c|c|c|c|c|}
\hline Analyte (Method) & Units & $\begin{array}{c}1^{\text {st }} \\
\text { Replicate } \\
\end{array}$ & $\begin{array}{c}2^{\text {nd }} \\
\text { Replicate } \\
\end{array}$ & $\begin{array}{c}3^{\text {rd }} \\
\text { Replicate } \\
\end{array}$ & Average & \%RSD & Blank \\
\hline Gd (IE) & $\mathrm{mg} / \mathrm{L}$ & $<6.8 \mathrm{E}+00$ & $<6.9 \mathrm{E}+00$ & $<6.9 \mathrm{E}+00$ & - & - & $<6.9 \mathrm{E}+00$ \\
\hline K (IE) & $\mathrm{mg} / \mathrm{L}$ & $1.23 \mathrm{E}+02$ & $1.33 \mathrm{E}+02$ & $1.29 \mathrm{E}+02$ & $1.29 \mathrm{E}+02$ & $4.0 \%$ & $<9.5 \mathrm{E}+01$ \\
\hline La (IE) & $\mathrm{mg} / \mathrm{L}$ & $<8.4 \mathrm{E}+00$ & $<8.4 \mathrm{E}+00$ & $<8.4 \mathrm{E}+00$ & - & - & $<8.4 \mathrm{E}+00$ \\
\hline Li (IE) & $\mathrm{mg} / \mathrm{L}$ & $<5.8 \mathrm{E}+00$ & $<5.8 \mathrm{E}+00$ & $<5.8 \mathrm{E}+00$ & - & - & $<5.8 \mathrm{E}+00$ \\
\hline Mg (IE) & $\mathrm{mg} / \mathrm{L}$ & $<6.8 \mathrm{E}-01$ & $<6.8 \mathrm{E}-01$ & $<6.8 \mathrm{E}-01$ & - & - & $<6.8 \mathrm{E}-01$ \\
\hline $\mathrm{Mn}$ (IE) & $\mathrm{mg} / \mathrm{L}$ & $<4.4 \mathrm{E}-01$ & $<4.4 \mathrm{E}-01$ & $<4.4 \mathrm{E}-01$ & - & - & $<4.4 \mathrm{E}-01$ \\
\hline Mo (IE) & $\mathrm{mg} / \mathrm{L}$ & $<6.3 \mathrm{E}+00$ & $<6.3 \mathrm{E}+00$ & $<6.3 \mathrm{E}+00$ & - & - & $<6.3 \mathrm{E}+00$ \\
\hline $\mathrm{Na}$ (IE) & $\mathrm{mg} / \mathrm{L}$ & $9.55 \mathrm{E}+04$ & $9.62 \mathrm{E}+04$ & $9.56 \mathrm{E}+04$ & $9.58 \mathrm{E}+04$ & $0.4 \%$ & $<5.5 \mathrm{E}+01$ \\
\hline $\mathrm{Ni}$ (IE) & $\mathrm{mg} / \mathrm{L}$ & $<4.5 \mathrm{E}+00$ & $<4.5 \mathrm{E}+00$ & $<4.5 \mathrm{E}+00$ & - & - & $<4.6 \mathrm{E}+00$ \\
\hline $\mathrm{P}$ (IE) & $\mathrm{mg} / \mathrm{L}$ & $<2.9 \mathrm{E}+01$ & $<2.9 \mathrm{E}+01$ & $<2.9 \mathrm{E}+01$ & - & - & $<2.9 \mathrm{E}+01$ \\
\hline $\mathrm{Pb}$ (IE) & $\mathrm{mg} / \mathrm{L}$ & $<1.6 \mathrm{E}+01$ & $<1.6 \mathrm{E}+01$ & $<1.6 \mathrm{E}+01$ & - & - & $<1.6 \mathrm{E}+01$ \\
\hline S (IE) & $\mathrm{mg} / \mathrm{L}$ & $6.00 \mathrm{E}+02$ & $5.88 \mathrm{E}+02$ & $5.93 \mathrm{E}+02$ & $5.94 \mathrm{E}+02$ & $1.1 \%$ & $<2.3 \mathrm{E}+02$ \\
\hline Sb (IE) & $\mathrm{mg} / \mathrm{L}$ & $<3.1 \mathrm{E}+01$ & $<3.1 \mathrm{E}+01$ & $<3.1 \mathrm{E}+01$ & - & - & $<3.1 \mathrm{E}+01$ \\
\hline Si (IE) & $\mathrm{mg} / \mathrm{L}$ & $<5.1 \mathrm{E}+01$ & $<5.1 \mathrm{E}+01$ & $<5.1 \mathrm{E}+01$ & - & - & $<5.2 \mathrm{E}+01$ \\
\hline Sn (IE) & $\mathrm{mg} / \mathrm{L}$ & $<9.3 \mathrm{E}+01$ & $<9.3 \mathrm{E}+01$ & $<9.3 \mathrm{E}+01$ & - & - & $<9.3 \mathrm{E}+01$ \\
\hline Sr (IE) & $\mathrm{mg} / \mathrm{L}$ & $<8.2 \mathrm{E}+00$ & $<8.3 \mathrm{E}+00$ & $<8.3 \mathrm{E}+00$ & - & - & $<8.3 \mathrm{E}+00$ \\
\hline Ti (IE) & $\mathrm{mg} / \mathrm{L}$ & $<3.7 \mathrm{E}+00$ & $<3.7 \mathrm{E}+00$ & $<3.7 \mathrm{E}+00$ & - & - & $<3.7 \mathrm{E}+00$ \\
\hline U (IE) & $\mathrm{mg} / \mathrm{L}$ & $<3.8 \mathrm{E}+02$ & $<3.8 \mathrm{E}+02$ & $<3.8 \mathrm{E}+02$ & - & - & $<3.8 \mathrm{E}+02$ \\
\hline V (IE) & $\mathrm{mg} / \mathrm{L}$ & $<3.6 \mathrm{E}+00$ & $<3.6 \mathrm{E}+00$ & $<3.6 \mathrm{E}+00$ & - & - & $<3.6 \mathrm{E}+00$ \\
\hline $\mathrm{Zn}$ (IE) & $\mathrm{mg} / \mathrm{L}$ & $<2.8 \mathrm{E}+00$ & $<2.8 \mathrm{E}+00$ & $<2.8 \mathrm{E}+00$ & - & - & $<2.8 \mathrm{E}+00$ \\
\hline Zr (IE) & $\mathrm{mg} / \mathrm{L}$ & $<2.2 \mathrm{E}+00$ & $<2.2 \mathrm{E}+00$ & $<2.2 \mathrm{E}+00$ & - & - & $<2.3 \mathrm{E}+00$ \\
\hline
\end{tabular}

The As-Received Tank 11 Supernate was not filtered prior to submitting samples for analysis at the request of the customer. 
WSRC-STI-2008-00227, REV. 0

Table 3-4. Composition of the As-Received Tank 11H Supernate (Continued)

\begin{tabular}{|c|c|c|c|c|c|c|c|}
\hline Analyte (Method) & Units & $\begin{array}{c}1^{\text {st }} \\
\text { Replicate } \\
\end{array}$ & $\begin{array}{c}2^{\text {nd }} \\
\text { Replicate }\end{array}$ & $\begin{array}{c}3^{\text {rd }} \\
\text { Replicate } \\
\end{array}$ & Average & \%RSD & Blank \\
\hline $\mathrm{Hg}(\mathrm{CV})$ & $\mathrm{mg} / \mathrm{L}$ & $1.80 \mathrm{E}+02$ & $1.80 \mathrm{E}+02$ & $1.80 \mathrm{E}+02$ & $1.80 \mathrm{E}+02$ & $0.2 \%$ & $<1.1 \mathrm{E}+00$ \\
\hline${ }^{232} \mathrm{U}(\mathrm{SA})$ & $\mathrm{mg} / \mathrm{L}$ & $<4.5 \mathrm{E}-07$ & $<2.7 \mathrm{E}-07$ & $<3.3 \mathrm{E}-07$ & - & - & $<2.0 \mathrm{E}-07$ \\
\hline${ }^{233} \mathrm{U}$ (IM) & $\mathrm{mg} / \mathrm{L}$ & $<2.0 \mathrm{E}-02$ & $<2.0 \mathrm{E}-02$ & $<2.0 \mathrm{E}-02$ & - & - & $<2.0 \mathrm{E}-02$ \\
\hline${ }^{234} \mathrm{U}$ (IM) & $\mathrm{mg} / \mathrm{L}$ & $<3.9 \mathrm{E}-02$ & $<4.0 \mathrm{E}-02$ & $<3.9 \mathrm{E}-02$ & - & - & $<4.0 \mathrm{E}-02$ \\
\hline${ }^{235} \mathrm{U}$ (IM) & $\mathrm{mg} / \mathrm{L}$ & $5.80 \mathrm{E}-02$ & 4.46E-02 & 4.65E-02 & 4.97E-02 & $15 \%$ & $<2.0 \mathrm{E}-02$ \\
\hline${ }^{236} \mathrm{U}$ (IM) & $\mathrm{mg} / \mathrm{L}$ & $<2.0 \mathrm{E}-02$ & $<2.0 \mathrm{E}-02$ & $<2.0 \mathrm{E}-02$ & - & - & $<2.0 \mathrm{E}-02$ \\
\hline${ }^{238} \mathrm{U}$ (IM) & $\mathrm{mg} / \mathrm{L}$ & $5.07 \mathrm{E}+00$ & 4.17E+00 & $4.99 \mathrm{E}+00$ & $4.74 \mathrm{E}+00$ & $11 \%$ & $<4.0 \mathrm{E}-02$ \\
\hline${ }^{137 m} \mathrm{Ba}(\mathrm{SG})$ & $\mu \mathrm{Ci} / \mathrm{mL}$ & $1.44 \mathrm{E}+01$ & $1.41 \mathrm{E}+01$ & $1.38 \mathrm{E}+01$ & $1.41 \mathrm{E}+01$ & $2.3 \%$ & $<7.2 \mathrm{E}-04$ \\
\hline${ }^{134}$ Cs (SG) & $\mu \mathrm{Ci} / \mathrm{mL}$ & $<1.7 \mathrm{E}-03$ & $<1.6 \mathrm{E}-03$ & $<1.7 \mathrm{E}-03$ & - & - & $<6.7 \mathrm{E}-04$ \\
\hline${ }^{137} \mathrm{Cs}$ (SG) & $\mu \mathrm{Ci} / \mathrm{mL}$ & $1.53 \mathrm{E}+01$ & $1.49 \mathrm{E}+01$ & $1.46 \mathrm{E}+01$ & $1.49 \mathrm{E}+01$ & $2.3 \%$ & $<7.6 \mathrm{E}-04$ \\
\hline${ }^{106} \mathrm{Ru}$ (SG) & $\mu \mathrm{Ci} / \mathrm{mL}$ & $<4.7 \mathrm{E}-02$ & $<4.6 \mathrm{E}-02$ & $<4.6 \mathrm{E}-02$ & - & - & $<4.8 \mathrm{E}-03$ \\
\hline${ }^{154} \mathrm{Eu}$ (SG) & $\mu \mathrm{Ci} / \mathrm{mL}$ & $<3.6 \mathrm{E}-03$ & $<3.5 \mathrm{E}-03$ & $<3.0 \mathrm{E}-03$ & - & - & $<9.2 \mathrm{E}-04$ \\
\hline${ }^{155} \mathrm{Eu}(\mathrm{SG})$ & $\mu \mathrm{Ci} / \mathrm{mL}$ & $<1.7 \mathrm{E}-02$ & $<1.7 \mathrm{E}-02$ & $<1.7 \mathrm{E}-02$ & - & - & $<1.7 \mathrm{E}-03$ \\
\hline${ }^{60}$ Co (SG) & $\mu \mathrm{Ci} / \mathrm{mL}$ & $<8.2 \mathrm{E}-04$ & $<8.2 \mathrm{E}-04$ & $<9.2 \mathrm{E}-04$ & - & - & $<7.5 \mathrm{E}-04$ \\
\hline${ }^{126} \mathrm{Sn}(\mathrm{SG})$ & $\mu \mathrm{Ci} / \mathrm{mL}$ & $<1.6 \mathrm{E}-02$ & $<9.0 \mathrm{E}-03$ & $<1.5 \mathrm{E}-02$ & - & - & $<1.4 \mathrm{E}-03$ \\
\hline${ }^{126} \mathrm{Sb}(\mathrm{SG})$ & $\mu \mathrm{Ci} / \mathrm{mL}$ & $<2.0 \mathrm{E}-03$ & $<1.9 \mathrm{E}-03$ & $<2.0 \mathrm{E}-03$ & - & - & $<6.5 \mathrm{E}-04$ \\
\hline${ }^{125} \mathrm{Sb}$ (SG) & $\mu \mathrm{Ci} / \mathrm{mL}$ & $<3.8 \mathrm{E}-02$ & $<3.8 \mathrm{E}-02$ & $<3.7 \mathrm{E}-02$ & - & - & $<1.9 \mathrm{E}-03$ \\
\hline${ }^{144} \mathrm{Ce}$ (SG) & $\mu \mathrm{Ci} / \mathrm{mL}$ & $<4.3 \mathrm{E}-02$ & $<4.2 \mathrm{E}-02$ & $<4.2 \mathrm{E}-02$ & - & - & $<3.5 \mathrm{E}-03$ \\
\hline${ }^{241} \mathrm{Am}$ (SG) & $\mu \mathrm{Ci} / \mathrm{mL}$ & $<4.1 \mathrm{E}-02$ & $<4.1 \mathrm{E}-02$ & $<4.1 \mathrm{E}-02$ & - & - & $<3.1 \mathrm{E}-03$ \\
\hline
\end{tabular}

The As-Received Tank 11 Supernate was not filtered prior to submitting samples for analysis at the request of the customer. 
WSRC-STI-2008-00227, REV. 0

Table 3-4. Composition of the As-Received Tank 11H Supernate (Continued)

\begin{tabular}{|c|c|c|c|c|c|c|c|}
\hline Analyte (Method) & Units & $\begin{array}{c}1^{\text {st }} \\
\text { Replicate } \\
\end{array}$ & $\begin{array}{c}2^{\text {nd }} \\
\text { Replicate }\end{array}$ & $\begin{array}{c}3^{\text {rd }} \\
\text { Replicate } \\
\end{array}$ & Average & $\%$ RSD & Blank \\
\hline${ }^{99}$ Tc (IM) & $\mu \mathrm{Ci} / \mathrm{mL}$ & 7.35E-03 & 5.95E-03 & 6.06E-03 & $6.45 \mathrm{E}-03$ & $12 \%$ & $<8.4 \mathrm{E}-04$ \\
\hline${ }^{237} \mathrm{~Np}$ (IM) & $\mu \mathrm{Ci} / \mathrm{mL}$ & $<1.4 \mathrm{E}-05$ & $<1.4 \mathrm{E}-05$ & $<1.4 \mathrm{E}-05$ & - & - & $<1.4 \mathrm{E}-05$ \\
\hline${ }^{239} \mathrm{Pu}$ (IM) & $\mu \mathrm{Ci} / \mathrm{mL}$ & $<3.6 \mathrm{E}-03$ & $<3.6 \mathrm{E}-03$ & $<3.6 \mathrm{E}-03$ & - & - & $<3.6 \mathrm{E}-03$ \\
\hline${ }^{240} \mathrm{Pu}(\mathrm{IM})$ & $\mu \mathrm{Ci} / \mathrm{mL}$ & $<4.4 \mathrm{E}-03$ & $<4.5 \mathrm{E}-03$ & $<4.4 \mathrm{E}-03$ & - & - & $<4.5 \mathrm{E}-03$ \\
\hline${ }^{238} \mathrm{Pu}$ (SA) & $\mu \mathrm{Ci} / \mathrm{mL}$ & 4.66E-02 & 5.12E-02 & 3.94E-02 & 4.57E-02 & $13 \%$ & $<5.9 \mathrm{E}-05$ \\
\hline${ }^{239 / 240} \mathrm{Pu}(\mathrm{SA})$ & $\mu \mathrm{Ci} / \mathrm{mL}$ & $1.84 \mathrm{E}-03$ & $2.17 \mathrm{E}-03$ & $1.65 \mathrm{E}-03$ & $1.88 \mathrm{E}-03$ & $14 \%$ & $<8.9 \mathrm{E}-05$ \\
\hline${ }^{241} \mathrm{Pu}(\mathrm{SA})$ & $\mu \mathrm{Ci} / \mathrm{mL}$ & $1.70 \mathrm{E}-02$ & 1.86E-02 & 1.47E-02 & $1.68 \mathrm{E}-02$ & $12 \%$ & $<4.2 \mathrm{E}-04$ \\
\hline${ }^{90} \mathrm{Sr}(\mathrm{SL})$ & $\mu \mathrm{Ci} / \mathrm{mL}$ & 6.38E-01 & $6.54 \mathrm{E}-01$ & $6.75 \mathrm{E}-01$ & $6.56 \mathrm{E}-01$ & $2.8 \%$ & $<2.1 \mathrm{E}-04$ \\
\hline${ }^{79}$ Se (SG) & $\mu \mathrm{Ci} / \mathrm{mL}$ & $<6.4 \mathrm{E}-06$ & $<6.9 \mathrm{E}-06$ & $<9.3 \mathrm{E}-06$ & - & - & - \\
\hline${ }^{129} \mathrm{I}(\mathrm{SG})$ & $\mu \mathrm{Ci} / \mathrm{mL}$ & $2.44 \mathrm{E}-05$ & $3.01 \mathrm{E}-05$ & $3.07 \mathrm{E}-05$ & $2.84 \mathrm{E}-05$ & $12 \%$ & - \\
\hline Total Alpha (SL) & $\mu \mathrm{Ci} / \mathrm{mL}$ & $<1.7 \mathrm{E}-01$ & $<1.2 \mathrm{E}-01$ & $<1.2 \mathrm{E}-01$ & - & - & $<1.4 \mathrm{E}-01$ \\
\hline Total Beta (SL) & $\mu \mathrm{Ci} / \mathrm{mL}$ & $1.86 \mathrm{E}+01$ & $1.89 \mathrm{E}+01$ & $1.89 \mathrm{E}+01$ & $1.88 \mathrm{E}+01$ & $0.8 \%$ & $<2.4 \mathrm{E}-01$ \\
\hline
\end{tabular}

The As-Received Tank 11 Supernate was not filtered prior to submitting samples for analysis at the request of the customer. 
WSRC-STI-2008-00227, REV. 0

Table 3-5. Composition of the As-Received Tank 51H Filtered Supernate

\begin{tabular}{|c|c|c|c|c|c|c|c|}
\hline Analyte (Method) & Units & $\begin{array}{c}\mathbf{1}^{\text {st }} \\
\text { Replicate } \\
\end{array}$ & $\begin{array}{c}2^{\text {nd }} \\
\text { Replicate } \\
\end{array}$ & $\begin{array}{c}3^{\text {rd }} \\
\text { Replicate } \\
\end{array}$ & Average & \%RSD & Blank \\
\hline $\mathrm{NO}_{3}^{-}$(IC) & M & $3.51 \mathrm{E}-01 *$ & $1.98 \mathrm{E}-01$ & $1.84 \mathrm{E}-01$ & $1.91 \mathrm{E}-01$ & $5.0 \%$ & $<8.1 \mathrm{E}-03$ \\
\hline $\mathrm{NO}_{2}^{-}$(IC) & M & 3.58E-01 & 3.79E-01 & $3.52 \mathrm{E}-01$ & 3.63E-01 & $3.9 \%$ & $<1.1 \mathrm{E}-02$ \\
\hline $\mathrm{PO}_{4}^{3-}$ (IC) & M & $<1.3 \mathrm{E}-02$ & $<1.3 \mathrm{E}-02$ & $<1.3 \mathrm{E}-02$ & - & - & $<1.3 \mathrm{E}-02$ \\
\hline $\mathrm{SO}_{4}{ }^{2-}$ (IC) & $\mathrm{M}$ & $1.81 \mathrm{E}-02$ & $1.75 \mathrm{E}-02$ & $1.80 \mathrm{E}-02$ & $1.79 \mathrm{E}-02$ & $1.7 \%$ & $<5.2 \mathrm{E}-03$ \\
\hline $\mathrm{C}_{2} \mathrm{O}_{4}{ }^{2-}$ (IC) & M & $<5.8 \mathrm{E}-03$ & $<5.8 \mathrm{E}-03$ & $<5.8 \mathrm{E}-03$ & - & - & $<5.7 \mathrm{E}-03$ \\
\hline $\mathrm{Cl}^{-}$(IC) & $\mathrm{M}$ & 2.89E-02 & 2.91E-02 & 2.93E-02 & 2.91E-02 & $0.8 \%$ & $<1.4 \mathrm{E}-02$ \\
\hline $\mathrm{F}^{-}(\mathrm{IC})$ & M & $<2.7 \mathrm{E}-02$ & $<2.7 \mathrm{E}-02$ & $<2.7 \mathrm{E}-02$ & - & - & $<2.7 \mathrm{E}-02$ \\
\hline $\mathrm{CHO}_{2}^{-}$(IC) & $\mathrm{M}$ & $<1.1 \mathrm{E}-02$ & $<1.1 \mathrm{E}-02$ & $<1.1 \mathrm{E}-02$ & - & - & $<1.1 \mathrm{E}-02$ \\
\hline $\mathrm{OH}_{\text {free }}^{-}(\mathrm{TH})$ & $\mathrm{M}$ & $3.15 \mathrm{E}+00$ & $3.10 \mathrm{E}+00$ & $3.07 \mathrm{E}+00$ & $3.11 \mathrm{E}+00$ & $1.3 \%$ & - \\
\hline $\mathrm{CO}_{3}{ }^{2-}$ (TIC) & $\mathrm{M}$ & $1.07 \mathrm{E}-01$ & $1.60 \mathrm{E}-01$ & $1.14 \mathrm{E}-01$ & $1.27 \mathrm{E}-01$ & $23 \%$ & $3.27 \mathrm{E}-03$ \\
\hline Ag (IE) & $\mathrm{mg} / \mathrm{L}$ & $<3.0 \mathrm{E}+00$ & $<3.0 \mathrm{E}+00$ & $<3.0 \mathrm{E}+00$ & - & - & $<2.9 \mathrm{E}+00$ \\
\hline Al (IE) & $\mathrm{mg} / \mathrm{L}$ & $1.11 \mathrm{E}+04$ & $1.12 \mathrm{E}+04$ & $1.12 \mathrm{E}+04$ & $1.12 \mathrm{E}+04$ & $0.4 \%$ & $<1.3 \mathrm{E}+01$ \\
\hline B (IE) & $\mathrm{mg} / \mathrm{L}$ & $<2.6 \mathrm{E}+00$ & $<2.5 \mathrm{E}+00$ & $<2.6 \mathrm{E}+00$ & - & - & $<2.5 \mathrm{E}+00$ \\
\hline Ba (IE) & $\mathrm{mg} / \mathrm{L}$ & $<6.4 \mathrm{E}-01$ & $<6.4 \mathrm{E}-01$ & $<6.4 \mathrm{E}-01$ & - & - & $<6.3 \mathrm{E}-01$ \\
\hline Be (IE) & $\mathrm{mg} / \mathrm{L}$ & $<1.1 \mathrm{E}-01$ & $<1.1 \mathrm{E}-01$ & $<1.1 \mathrm{E}-01$ & - & - & $<1.1 \mathrm{E}-01$ \\
\hline $\mathrm{Ca}$ (IE) & $\mathrm{mg} / \mathrm{L}$ & $<8.1 \mathrm{E}+00$ & $<8.1 \mathrm{E}+00$ & $<8.1 \mathrm{E}+00$ & - & - & $<8.1 \mathrm{E}+00$ \\
\hline Cd (IE) & $\mathrm{mg} / \mathrm{L}$ & $<8.4 \mathrm{E}-01$ & $<8.3 \mathrm{E}-01$ & $<8.4 \mathrm{E}-01$ & - & - & $<8.3 \mathrm{E}-01$ \\
\hline Ce (IE) & $\mathrm{mg} / \mathrm{L}$ & $<3.3 \mathrm{E}+01$ & $<3.2 \mathrm{E}+01$ & $<3.3 \mathrm{E}+01$ & - & - & $<3.2 \mathrm{E}+01$ \\
\hline $\mathrm{Cr}$ (IE) & $\mathrm{mg} / \mathrm{L}$ & $2.61 \mathrm{E}+01$ & $2.60 \mathrm{E}+01$ & $2.60 \mathrm{E}+01$ & $2.60 \mathrm{E}+01$ & $0.1 \%$ & $<1.0 \mathrm{E}+00$ \\
\hline $\mathrm{Cu}$ (IE) & $\mathrm{mg} / \mathrm{L}$ & $<1.7 \mathrm{E}+00$ & $<1.7 \mathrm{E}+00$ & $<1.7 \mathrm{E}+00$ & - & - & $<1.7 \mathrm{E}+00$ \\
\hline Fe (IE) & $\mathrm{mg} / \mathrm{L}$ & $2.52 \mathrm{E}+00$ & $<1.6 \mathrm{E}+00$ & $<1.6 \mathrm{E}+00$ & $2.52 \mathrm{E}+00 * *$ & - & $<1.6 \mathrm{E}+00$ \\
\hline
\end{tabular}

* The nitrate value measured for the first replicate was dropped from calculation of the average since the value was more than $50 \%$ greater than the average of the other two replicates.

** Only a single value was above the detection limit. 
WSRC-STI-2008-00227, REV. 0

Table 3-5. Composition of the As-Received Tank 51H Filtered Supernate (Continued)

\begin{tabular}{|c|c|c|c|c|c|c|c|}
\hline Analyte (Method) & Units & $\begin{array}{c}1^{\text {st }} \\
\text { Replicate } \\
\end{array}$ & $\begin{array}{c}2^{\text {nd }} \\
\text { Replicate } \\
\end{array}$ & $\begin{array}{c}3^{\text {rd }} \\
\text { Replicate } \\
\end{array}$ & Average & \%RSD & Blank \\
\hline Gd (IE) & $\mathrm{mg} / \mathrm{L}$ & $<3.5 \mathrm{E}+00$ & $<3.5 \mathrm{E}+00$ & $<3.5 \mathrm{E}+00$ & - & - & $<3.5 \mathrm{E}+00$ \\
\hline K (IE) & $\mathrm{mg} / \mathrm{L}$ & $1.54 \mathrm{E}+02$ & $1.66 \mathrm{E}+02$ & $1.61 \mathrm{E}+02$ & $1.60 \mathrm{E}+02$ & $3.7 \%$ & $<4.8 \mathrm{E}+01$ \\
\hline La (IE) & $\mathrm{mg} / \mathrm{L}$ & $<4.3 \mathrm{E}+00$ & $<4.3 \mathrm{E}+00$ & $<4.3 \mathrm{E}+00$ & - & - & $<4.3 \mathrm{E}+00$ \\
\hline Li (IE) & $\mathrm{mg} / \mathrm{L}$ & $<3.0 \mathrm{E}+00$ & $<3.0 \mathrm{E}+00$ & $<3.0 \mathrm{E}+00$ & - & - & $<3.0 \mathrm{E}+00$ \\
\hline Mg (IE) & $\mathrm{mg} / \mathrm{L}$ & $<3.5 \mathrm{E}-01$ & $<3.5 \mathrm{E}-01$ & $<3.5 \mathrm{E}-01$ & - & - & $<3.5 \mathrm{E}-01$ \\
\hline Mn (IE) & $\mathrm{mg} / \mathrm{L}$ & $<2.3 \mathrm{E}-01$ & $<2.2 \mathrm{E}-01$ & $<2.2 \mathrm{E}-01$ & - & - & $<2.2 \mathrm{E}-01$ \\
\hline Mo (IE) & $\mathrm{mg} / \mathrm{L}$ & $<3.2 \mathrm{E}+00$ & $<3.2 \mathrm{E}+00$ & $<3.2 \mathrm{E}+00$ & - & - & $<3.2 \mathrm{E}+00$ \\
\hline $\mathrm{Na}$ (IE) & $\mathrm{mg} / \mathrm{L}$ & $9.27 \mathrm{E}+04$ & $9.31 E+04$ & $9.37 \mathrm{E}+04$ & $9.32 E+04$ & $0.5 \%$ & $<2.8 \mathrm{E}+01$ \\
\hline $\mathrm{Ni}$ (IE) & $\mathrm{mg} / \mathrm{L}$ & $<2.3 \mathrm{E}+00$ & $<2.3 \mathrm{E}+00$ & $<2.3 \mathrm{E}+00$ & - & - & $<2.3 \mathrm{E}+00$ \\
\hline $\mathrm{P}$ (IE) & $\mathrm{mg} / \mathrm{L}$ & $2.45 \mathrm{E}+01$ & $2.52 \mathrm{E}+01$ & $2.20 \mathrm{E}+01$ & $2.39 \mathrm{E}+01$ & $7.1 \%$ & $<1.5 \mathrm{E}+01$ \\
\hline $\mathrm{Pb}$ (IE) & $\mathrm{mg} / \mathrm{L}$ & $<8.4 \mathrm{E}+00$ & $<8.4 \mathrm{E}+00$ & $<8.4 \mathrm{E}+00$ & - & - & $<8.3 \mathrm{E}+00$ \\
\hline S (IE) & $\mathrm{mg} / \mathrm{L}$ & $5.79 \mathrm{E}+02$ & $5.96 \mathrm{E}+02$ & $5.99 \mathrm{E}+02$ & $5.92 \mathrm{E}+02$ & $1.8 \%$ & $<1.9 \mathrm{E}+02$ \\
\hline $\mathrm{Sb}$ (IE) & $\mathrm{mg} / \mathrm{L}$ & $<1.6 \mathrm{E}+01$ & $<1.6 \mathrm{E}+01$ & $<1.6 \mathrm{E}+01$ & - & - & $<1.6 \mathrm{E}+01$ \\
\hline Si (IE) & $\mathrm{mg} / \mathrm{L}$ & $<2.6 \mathrm{E}+01$ & $<2.6 \mathrm{E}+01$ & $<2.6 \mathrm{E}+01$ & - & - & $<2.6 \mathrm{E}+01$ \\
\hline Sn (IE) & $\mathrm{mg} / \mathrm{L}$ & $<4.8 \mathrm{E}+01$ & $<4.8 \mathrm{E}+01$ & $<4.8 \mathrm{E}+01$ & - & - & $<4.7 \mathrm{E}+01$ \\
\hline Sr (IE) & $\mathrm{mg} / \mathrm{L}$ & $<4.3 \mathrm{E}+00$ & $<4.3 \mathrm{E}+00$ & $<4.3 \mathrm{E}+00$ & - & - & $<4.2 \mathrm{E}+00$ \\
\hline Ti (IE) & $\mathrm{mg} / \mathrm{L}$ & $<1.9 \mathrm{E}+00$ & $<1.9 \mathrm{E}+00$ & $<1.9 \mathrm{E}+00$ & - & - & $<1.9 \mathrm{E}+00$ \\
\hline U (IE) & $\mathrm{mg} / \mathrm{L}$ & $<1.6 \mathrm{E}+02$ & $<1.6 \mathrm{E}+02$ & $<1.6 \mathrm{E}+02$ & - & - & $<1.6 \mathrm{E}+02$ \\
\hline $\mathrm{V}$ (IE) & $\mathrm{mg} / \mathrm{L}$ & $<1.9 \mathrm{E}+00$ & $<1.8 \mathrm{E}+00$ & $<1.9 \mathrm{E}+00$ & - & - & $<1.8 \mathrm{E}+00$ \\
\hline $\mathrm{Zn}$ (IE) & $\mathrm{mg} / \mathrm{L}$ & $<1.4 \mathrm{E}+00$ & $<1.4 \mathrm{E}+00$ & $<1.4 \mathrm{E}+00$ & - & - & $<1.4 \mathrm{E}+00$ \\
\hline Zr (IE) & $\mathrm{mg} / \mathrm{L}$ & $<1.2 \mathrm{E}+00$ & $<1.2 \mathrm{E}+00$ & $<1.2 \mathrm{E}+00$ & - & - & $<1.1 \mathrm{E}+00$ \\
\hline
\end{tabular}


WSRC-STI-2008-00227, REV. 0

Table 3-5. Composition of the As-Received Tank 51H Filtered Supernate (Continued)

\begin{tabular}{|c|c|c|c|c|c|c|c|}
\hline Analyte (Method) & Units & $\begin{array}{c}\mathbf{1}^{\text {st }} \\
\text { Replicate }\end{array}$ & $\begin{array}{c}2^{\text {nd }} \\
\text { Replicate } \\
\end{array}$ & $\begin{array}{c}3^{\text {rd }} \\
\text { Replicate }\end{array}$ & Average & \%RSD & Blank \\
\hline $\mathrm{Hg}(\mathrm{CV})$ & $\mathrm{mg} / \mathrm{L}$ & $1.25 \mathrm{E}+02$ & $1.27 \mathrm{E}+02$ & $1.25 \mathrm{E}+02$ & $1.26 \mathrm{E}+02$ & $0.8 \%$ & $<1.1 \mathrm{E}+00$ \\
\hline${ }^{232} \mathrm{U}(\mathrm{SA})$ & $\mathrm{mg} / \mathrm{L}$ & $<2.2 \mathrm{E}-07$ & $<1.7 \mathrm{E}-07$ & $<6.2 \mathrm{E}-07$ & - & - & $<4.5 \mathrm{E}-08$ \\
\hline${ }^{233} \mathrm{U}(\mathrm{IM})$ & $\mathrm{mg} / \mathrm{L}$ & $<1.0 \mathrm{E}-01$ & $<1.0 \mathrm{E}-01$ & $<1.0 \mathrm{E}-01$ & - & - & $<1.0 \mathrm{E}-01$ \\
\hline${ }^{234} \mathrm{U}$ (IM) & $\mathrm{mg} / \mathrm{L}$ & $<1.0 \mathrm{E}-01$ & $<1.0 \mathrm{E}-01$ & $<1.0 \mathrm{E}-01$ & - & - & $<1.0 \mathrm{E}-01$ \\
\hline${ }^{235} \mathrm{U}(\mathrm{IM})$ & $\mathrm{mg} / \mathrm{L}$ & $<1.0 \mathrm{E}-01$ & $<1.0 \mathrm{E}-01$ & $<1.0 \mathrm{E}-01$ & - & - & $<1.0 \mathrm{E}-01$ \\
\hline${ }^{236} \mathrm{U}(\mathrm{IM})$ & $\mathrm{mg} / \mathrm{L}$ & $<1.0 \mathrm{E}-01$ & $<1.0 \mathrm{E}-01$ & $<1.0 \mathrm{E}-01$ & - & - & $<1.0 \mathrm{E}-01$ \\
\hline${ }^{238} \mathrm{U}$ (IM) & $\mathrm{mg} / \mathrm{L}$ & $1.81 \mathrm{E}+00$ & $1.75 \mathrm{E}+00$ & $1.82 \mathrm{E}+00$ & $1.79 \mathrm{E}+00$ & $2.3 \%$ & $<5.0 \mathrm{E}-01$ \\
\hline${ }^{137 m} \mathrm{Ba}(\mathrm{SG})$ & $\mu \mathrm{Ci} / \mathrm{mL}$ & $1.42 \mathrm{E}+01$ & $1.41 \mathrm{E}+01$ & $1.43 \mathrm{E}+01$ & $1.42 \mathrm{E}+01$ & $0.6 \%$ & $4.38 \mathrm{E}-04$ \\
\hline${ }^{134}$ Cs (SG) & $\mu \mathrm{Ci} / \mathrm{mL}$ & $<1.2 \mathrm{E}-03$ & $<1.1 \mathrm{E}-03$ & $<1.2 \mathrm{E}-03$ & - & - & $<5.3 \mathrm{E}-04$ \\
\hline${ }^{137} \mathrm{Cs}$ (SG) & $\mu \mathrm{Ci} / \mathrm{mL}$ & $1.50 \mathrm{E}+01$ & $1.49 \mathrm{E}+01$ & $1.51 \mathrm{E}+01$ & $1.50 \mathrm{E}+01$ & $0.6 \%$ & 4.63E-04 \\
\hline${ }^{106} \mathrm{Ru}$ (SG) & $\mu \mathrm{Ci} / \mathrm{mL}$ & $<3.3 \mathrm{E}-02$ & $<3.3 \mathrm{E}-02$ & $<3.3 \mathrm{E}-02$ & - & - & $<3.2 \mathrm{E}-03$ \\
\hline${ }^{154} \mathrm{Eu}$ (SG) & $\mu \mathrm{Ci} / \mathrm{mL}$ & $<2.1 \mathrm{E}-03$ & $<2.2 \mathrm{E}-03$ & $<2.4 \mathrm{E}-03$ & - & - & $<6.4 \mathrm{E}-04$ \\
\hline${ }^{155} \mathrm{Eu}(\mathrm{SG})$ & $\mu \mathrm{Ci} / \mathrm{mL}$ & $<9.0 \mathrm{E}-03$ & $<1.2 \mathrm{E}-02$ & $<1.2 \mathrm{E}-02$ & - & - & $<7.5 \mathrm{E}-04$ \\
\hline${ }^{60}$ Co (SG) & $\mu \mathrm{Ci} / \mathrm{mL}$ & $<5.8 \mathrm{E}-04$ & $<5.8 \mathrm{E}-04$ & $<6.3 \mathrm{E}-04$ & - & - & $<6.1 \mathrm{E}-04$ \\
\hline${ }^{126} \mathrm{Sn}(\mathrm{SG})$ & $\mu \mathrm{Ci} / \mathrm{mL}$ & $<7.9 \mathrm{E}-03$ & $<7.8 \mathrm{E}-03$ & $<1.1 \mathrm{E}-02$ & - & - & $<6.7 \mathrm{E}-04$ \\
\hline${ }^{126} \mathrm{Sb}(\mathrm{SG})$ & $\mu \mathrm{Ci} / \mathrm{mL}$ & $<1.4 \mathrm{E}-03$ & $<1.4 \mathrm{E}-03$ & $<1.5 \mathrm{E}-03$ & - & - & $<4.7 \mathrm{E}-04$ \\
\hline${ }^{125} \mathrm{Sb}$ (SG) & $\mu \mathrm{Ci} / \mathrm{mL}$ & $<2.7 \mathrm{E}-02$ & $<2.7 \mathrm{E}-02$ & $<2.7 \mathrm{E}-02$ & - & - & $<1.4 \mathrm{E}-03$ \\
\hline${ }^{144} \mathrm{Ce}$ (SG) & $\mu \mathrm{Ci} / \mathrm{mL}$ & $<3.0 \mathrm{E}-02$ & $<3.0 \mathrm{E}-02$ & $<3.1 \mathrm{E}-02$ & - & - & $<2.5 \mathrm{E}-03$ \\
\hline${ }^{241} \mathrm{Am}$ (SG) & $\mu \mathrm{Ci} / \mathrm{mL}$ & $<3.0 \mathrm{E}-02$ & $<3.0 \mathrm{E}-02$ & $<3.0 \mathrm{E}-02$ & - & - & $<2.3 \mathrm{E}-03$ \\
\hline
\end{tabular}


WSRC-STI-2008-00227, REV. 0

Table 3-5. Composition of the As-Received Tank 51H Filtered Supernate (Continued)

\begin{tabular}{|c|c|c|c|c|c|c|c|}
\hline Analyte (Method) & Units & $\begin{array}{c}1^{\text {st }} \\
\text { Replicate } \\
\end{array}$ & $\begin{array}{c}2^{\text {nd }} \\
\text { Replicate } \\
\end{array}$ & $\begin{array}{c}3^{\text {rd }} \\
\text { Replicate }\end{array}$ & Average & \%RSD & Blank \\
\hline${ }^{99}$ Tc (IM) & $\mu \mathrm{Ci} / \mathrm{mL}$ & 8.30E-03 & 7.12E-03 & $6.56 \mathrm{E}-03$ & 7.32E-03 & $12 \%$ & $2.62 \mathrm{E}-03$ \\
\hline${ }^{237} \mathrm{~Np}$ (IM) & $\mu \mathrm{Ci} / \mathrm{mL}$ & $<7.2 \mathrm{E}-05$ & $<7.2 \mathrm{E}-05$ & $<7.2 \mathrm{E}-05$ & - & - & $<7.1 \mathrm{E}-05$ \\
\hline${ }^{239} \mathrm{Pu}$ (IM) & $\mu \mathrm{Ci} / \mathrm{mL}$ & $<6.2 \mathrm{E}-03$ & $<6.2 \mathrm{E}-03$ & $<6.2 \mathrm{E}-03$ & - & - & $<6.2 \mathrm{E}-03$ \\
\hline${ }^{240} \mathrm{Pu}$ (IM) & $\mu \mathrm{Ci} / \mathrm{mL}$ & $<2.3 \mathrm{E}-02$ & $<2.3 \mathrm{E}-02$ & $<2.3 \mathrm{E}-02$ & - & - & $<2.3 \mathrm{E}-02$ \\
\hline${ }^{238} \mathrm{Pu}$ (SA) & $\mu \mathrm{Ci} / \mathrm{mL}$ & $1.03 \mathrm{E}-01$ & $1.03 \mathrm{E}-01$ & 1.19E-01 & $1.08 \mathrm{E}-01$ & $8.3 \%$ & $<9.2 \mathrm{E}-05$ \\
\hline${ }^{239 / 240} \mathrm{Pu}(\mathrm{SA})$ & $\mu \mathrm{Ci} / \mathrm{mL}$ & $5.14 \mathrm{E}-03$ & $4.76 \mathrm{E}-03$ & $5.50 \mathrm{E}-03$ & 5.13E-03 & $7.2 \%$ & $<3.7 \mathrm{E}-05$ \\
\hline${ }^{241} \mathrm{Pu}$ (SA) & $\mu \mathrm{Ci} / \mathrm{mL}$ & $<2.6 \mathrm{E}-02$ & $<2.7 \mathrm{E}-02$ & $<4.2 \mathrm{E}-02$ & - & - & $<1.6 \mathrm{E}-04$ \\
\hline${ }^{90} \mathrm{Sr}$ (SL) & $\mu \mathrm{Ci} / \mathrm{mL}$ & $3.78 \mathrm{E}-01$ & 3.82E-01 & 3.93E-01 & $3.84 \mathrm{E}-01$ & $2.1 \%$ & $<1.0 \mathrm{E}-04$ \\
\hline${ }^{79}$ Se (SG) & $\mu \mathrm{Ci} / \mathrm{mL}$ & $<1.2 \mathrm{E}-05$ & $<7.7 \mathrm{E}-06$ & $<1.6 \mathrm{E}-05$ & - & - & - \\
\hline${ }^{129} \mathrm{I}(\mathrm{SG})$ & $\mu \mathrm{Ci} / \mathrm{mL}$ & 7.57E-06 & $9.10 \mathrm{E}-06$ & 8.33E-06 & 8.33E-06 & $9.2 \%$ & - \\
\hline Total Alpha (SL) & $\mu \mathrm{Ci} / \mathrm{mL}$ & $<9.9 \mathrm{E}-02$ & $<1.0 \mathrm{E}-01$ & $<1.1 \mathrm{E}-01$ & & & $<9.5 \mathrm{E}-02$ \\
\hline Total Beta (SL) & $\mu \mathrm{Ci} / \mathrm{mL}$ & $1.88 \mathrm{E}+01$ & $1.88 \mathrm{E}+01$ & $1.85 \mathrm{E}+01$ & $1.87 \mathrm{E}+01$ & $1.1 \%$ & $<2.3 E-01$ \\
\hline
\end{tabular}


WSRC-STI-2008-00227, REV. 0

Table 3-6. Composition of the Aqua Regia Dissolution of Total Dried Solids from the As-Received Tank 51H Sludge

\begin{tabular}{|c|c|c|c|c|c|c|c|c|c|}
\hline Analyte (Method) & Units & $\begin{array}{c}\text { 1st } \\
\text { Replicate } \\
\end{array}$ & $\begin{array}{c}\text { 2nd } \\
\text { Replicate } \\
\end{array}$ & $\begin{array}{c}\text { 3rd } \\
\text { Replicate } \\
\end{array}$ & Average & \%RSD & Blank & $\begin{array}{l}\text { Average } \\
\text { Analyzed } \\
\text { Glass Std }\end{array}$ & $\begin{array}{c}\text { Glass Std } \\
\text { Composition }\end{array}$ \\
\hline Ag (IE) & $\mathrm{mg} / \mathrm{kg}$ & $<9.9 \mathrm{E}+02$ & $<9.8 \mathrm{E}+02$ & $<9.7 \mathrm{E}+02$ & - & - & $<9.6 \mathrm{E}+02$ & $<1.2 \mathrm{E}+03$ & - \\
\hline $\mathrm{Al}$ (IE) & $\mathrm{mg} / \mathrm{kg}$ & $7.92 \mathrm{E}+04$ & $8.20 \mathrm{E}+04$ & $7.98 \mathrm{E}+04$ & $8.03 E+04$ & $1.8 \%$ & $<1.3 \mathrm{E}+03$ & $2.39 \mathrm{E}+04$ & $2.50 \mathrm{E}+04$ \\
\hline $\mathrm{B}$ (IE) & $\mathrm{mg} / \mathrm{kg}$ & $<7.7 \mathrm{E}+02$ & $<7.6 \mathrm{E}+02$ & $<7.5 \mathrm{E}+02$ & - & - & $<7.5 \mathrm{E}+02$ & $2.50 \mathrm{E}+04$ & $2.69 \mathrm{E}+04$ \\
\hline Ва (IE) & $\mathrm{mg} / \mathrm{kg}$ & $3.84 \mathrm{E}+02$ & $3.91 \mathrm{E}+02$ & $3.87 \mathrm{E}+02$ & $3.87 \mathrm{E}+02$ & $1.0 \%$ & $<6.3 \mathrm{E}+01$ & $8.51 \mathrm{E}+02$ & $7.90 \mathrm{E}+02$ \\
\hline Be (IE) & $\mathrm{mg} / \mathrm{kg}$ & $<1.1 \mathrm{E}+01$ & $<1.1 \mathrm{E}+01$ & $<1.1 \mathrm{E}+01$ & - & - & $<1.1 \mathrm{E}+01$ & $1.98 \mathrm{E}+01$ & - \\
\hline $\mathrm{Ca}$ (IE) & $\mathrm{mg} / \mathrm{kg}$ & $6.65 \mathrm{E}+03$ & $6.82 E+03$ & $6.56 \mathrm{E}+03$ & $6.68 \mathrm{E}+03$ & $2.0 \%$ & $<9.8 \mathrm{E}+02$ & $1.08 \mathrm{E}+04$ & $1.02 \mathrm{E}+04$ \\
\hline Cd (IE) & $\mathrm{mg} / \mathrm{kg}$ & $2.23 \mathrm{E}+02$ & $2.33 \mathrm{E}+02$ & $2.23 \mathrm{E}+02$ & $2.26 \mathrm{E}+02$ & $2.4 \%$ & $<8.2 \mathrm{E}+01$ & $<9.9 \mathrm{E}+01$ & - \\
\hline Ce (IE) & $\mathrm{mg} / \mathrm{kg}$ & $<3.3 \mathrm{E}+03$ & $<3.3 \mathrm{E}+03$ & $<3.2 \mathrm{E}+03$ & - & - & $<3.2 \mathrm{E}+03$ & $<3.8 \mathrm{E}+03$ & - \\
\hline Cr (IE) & $\mathrm{mg} / \mathrm{kg}$ & $3.87 \mathrm{E}+02$ & $3.80 \mathrm{E}+02$ & $3.73 E+02$ & $3.80 \mathrm{E}+02$ & $1.8 \%$ & $<6.5 \mathrm{E}+01$ & $7.99 \mathrm{E}+02$ & $6.40 \mathrm{E}+02$ \\
\hline $\mathrm{Cu}$ (IE) & $\mathrm{mg} / \mathrm{kg}$ & $1.99 \mathrm{E}+02$ & $2.03 E+02$ & $2.08 \mathrm{E}+02$ & $2.03 E+02$ & $2.2 \%$ & $<1.7 \mathrm{E}+02$ & $<2.0 \mathrm{E}+02$ & $3.00 \mathrm{E}+01$ \\
\hline Fe (IE) & $\mathrm{mg} / \mathrm{kg}$ & $5.83 E+04$ & $6.07 E+04$ & $5.82 \mathrm{E}+04$ & $5.91 \mathrm{E}+04$ & $2.4 \%$ & $<1.6 \mathrm{E}+02$ & $1.01 \mathrm{E}+05$ & $9.79 \mathrm{E}+04$ \\
\hline Gd (IE) & $\mathrm{mg} / \mathrm{kg}$ & $<3.6 \mathrm{E}+02$ & $<3.6 \mathrm{E}+02$ & $<3.5 \mathrm{E}+02$ & - & - & $<3.5 \mathrm{E}+02$ & $<4.2 \mathrm{E}+02$ & - \\
\hline K (IE) & $\mathrm{mg} / \mathrm{kg}$ & $<4.9 \mathrm{E}+03$ & $<4.9 \mathrm{E}+03$ & $<4.8 \mathrm{E}+03$ & - & - & $<4.8 \mathrm{E}+03$ & $2.08 \mathrm{E}+04$ & $2.26 \mathrm{E}+04$ \\
\hline La (IE) & $\mathrm{mg} / \mathrm{kg}$ & $<4.4 \mathrm{E}+02$ & $<4.4 \mathrm{E}+02$ & $<4.3 \mathrm{E}+02$ & - & - & $<4.3 \mathrm{E}+02$ & $<5.1 \mathrm{E}+02$ & - \\
\hline Li (IE) & $\mathrm{mg} / \mathrm{kg}$ & $<3.0 \mathrm{E}+02$ & $<3.0 \mathrm{E}+02$ & $<3.0 \mathrm{E}+02$ & - & - & $<2.9 \mathrm{E}+02$ & $1.57 \mathrm{E}+04$ & $1.49 \mathrm{E}+04$ \\
\hline $\mathrm{Mg}$ (IE) & $\mathrm{mg} / \mathrm{kg}$ & $2.43 E+03$ & $2.53 E+03$ & $2.44 \mathrm{E}+03$ & $2.47 \mathrm{E}+03$ & $2.3 \%$ & $8.98 \mathrm{E}+01$ & $5.23 \mathrm{E}+03$ & $5.20 \mathrm{E}+03$ \\
\hline Mn (IE) & $\mathrm{mg} / \mathrm{kg}$ & $1.36 \mathrm{E}+04$ & $1.40 \mathrm{E}+04$ & $1.35 \mathrm{E}+04$ & $1.37 \mathrm{E}+04$ & $2.1 \%$ & $<9.6 \mathrm{E}+01$ & $1.43 \mathrm{E}+04$ & $1.46 \mathrm{E}+04$ \\
\hline Mo (IE) & $\mathrm{mg} / \mathrm{kg}$ & $<3.3 \mathrm{E}+02$ & $<3.3 \mathrm{E}+02$ & $<3.2 \mathrm{E}+02$ & - & - & $<3.2 \mathrm{E}+02$ & $<3.8 \mathrm{E}+02$ & - \\
\hline $\mathrm{Na}$ (IE) & $\mathrm{mg} / \mathrm{kg}$ & $3.03 E+05$ & $3.01 \mathrm{E}+05$ & $3.03 E+05$ & $3.03 E+05$ & $0.4 \%$ & $<2.8 \mathrm{E}+03$ & $8.48 \mathrm{E}+04$ & $8.52 E+04$ \\
\hline $\mathrm{Ni}$ (IE) & $\mathrm{mg} / \mathrm{kg}$ & $4.57 \mathrm{E}+03$ & $4.78 \mathrm{E}+03$ & $4.63 E+03$ & $4.66 \mathrm{E}+03$ & $2.4 \%$ & $<5.1 \mathrm{E}+02$ & $7.85 \mathrm{E}+03$ & $8.27 \mathrm{E}+03$ \\
\hline
\end{tabular}

Divide $\mathrm{mg} / \mathrm{kg}$ values by $1 \mathrm{E}+04$ to convert to wt $\%$ dried solids basis 
WSRC-STI-2008-00227, REV. 0

Table 3-6. Composition of the Aqua Regia Dissolution of Total Dried Solids from the As-Received Tank 51H Sludge (Continued)

\begin{tabular}{|c|c|c|c|c|c|c|c|c|c|}
\hline Analyte (Method) & Units & $\begin{array}{c}\text { 1st } \\
\text { Replicate } \\
\end{array}$ & $\begin{array}{c}\text { 2nd } \\
\text { Replicate } \\
\end{array}$ & $\begin{array}{c}\text { 3rd } \\
\text { Replicate } \\
\end{array}$ & Average & \%RSD & Blank & $\begin{array}{c}\text { Average } \\
\text { Analyzed } \\
\text { Glass Std }\end{array}$ & $\begin{array}{c}\text { Glass Std } \\
\text { Composition }\end{array}$ \\
\hline $\mathrm{P}$ (IE) & $\mathrm{mg} / \mathrm{kg}$ & $8.82 \mathrm{E}+02$ & $8.39 \mathrm{E}+02$ & $8.78 \mathrm{E}+02$ & $8.66 \mathrm{E}+02$ & $2.7 \%$ & $<7.5 \mathrm{E}+02$ & $9.31 \mathrm{E}+02$ & $1.10 \mathrm{E}+03$ \\
\hline $\mathrm{Pb}$ (IE) & $\mathrm{mg} / \mathrm{kg}$ & $<8.5 \mathrm{E}+02$ & $<8.5 \mathrm{E}+02$ & $<8.3 \mathrm{E}+02$ & - & - & $<8.3 \mathrm{E}+02$ & $<9.9 \mathrm{E}+02$ & - \\
\hline S (IE) & $\mathrm{mg} / \mathrm{kg}$ & $<1.2 \mathrm{E}+04$ & $<1.2 \mathrm{E}+04$ & $<1.2 \mathrm{E}+04$ & - & - & $<1.2 \mathrm{E}+04$ & $<1.4 \mathrm{E}+04$ & - \\
\hline Sb (IE) & $\mathrm{mg} / \mathrm{kg}$ & $<1.6 \mathrm{E}+03$ & $<1.6 \mathrm{E}+03$ & $<1.6 \mathrm{E}+03$ & - & - & $<1.6 \mathrm{E}+03$ & $<1.9 \mathrm{E}+03$ & - \\
\hline Sn (IE) & $\mathrm{mg} / \mathrm{kg}$ & $<4.8 \mathrm{E}+03$ & $<4.8 \mathrm{E}+03$ & $<4.7 \mathrm{E}+03$ & - & - & $<4.7 \mathrm{E}+03$ & $<5.6 \mathrm{E}+03$ & - \\
\hline Sr (IE) & $\mathrm{mg} / \mathrm{kg}$ & $<4.3 \mathrm{E}+02$ & $<4.3 \mathrm{E}+02$ & $<4.2 \mathrm{E}+02$ & - & - & $<4.2 \mathrm{E}+02$ & $<5.0 \mathrm{E}+02$ & $3.00 \mathrm{E}+01$ \\
\hline Ti (IE) & $\mathrm{mg} / \mathrm{kg}$ & $<1.9 \mathrm{E}+02$ & $<1.9 \mathrm{E}+02$ & $<1.9 \mathrm{E}+02$ & - & - & $<1.9 \mathrm{E}+02$ & $5.84 \mathrm{E}+03$ & $6.90 \mathrm{E}+03$ \\
\hline U (IE) & $\mathrm{mg} / \mathrm{kg}$ & $<2.0 \mathrm{E}+04$ & $<2.0 \mathrm{E}+04$ & $<1.9 \mathrm{E}+04$ & - & - & $<1.9 \mathrm{E}+04$ & $<2.3 \mathrm{E}+04$ & - \\
\hline $\mathrm{V}$ (IE) & $\mathrm{mg} / \mathrm{kg}$ & $<1.9 \mathrm{E}+02$ & $<1.9 \mathrm{E}+02$ & $<1.8 \mathrm{E}+02$ & - & - & $<1.8 \mathrm{E}+02$ & $<2.2 \mathrm{E}+02$ & - \\
\hline Zn (IE) & $\mathrm{mg} / \mathrm{kg}$ & $2.60 \mathrm{E}+02$ & $2.63 \mathrm{E}+02$ & $2.82 \mathrm{E}+02$ & $2.69 \mathrm{E}+02$ & $4.4 \%$ & $<1.4 \mathrm{E}+02$ & $2.06 \mathrm{E}+02$ & $1.60 \mathrm{E}+02$ \\
\hline $\mathrm{Zr}$ (IE) & $\mathrm{mg} / \mathrm{kg}$ & $8.12 \mathrm{E}+02$ & $8.34 \mathrm{E}+02$ & $8.18 \mathrm{E}+02$ & $8.21 \mathrm{E}+02$ & $1.4 \%$ & $<2.0 \mathrm{E}+02$ & $6.04 \mathrm{E}+02$ & $9.60 \mathrm{E}+02$ \\
\hline $\mathrm{Hg}(\mathrm{CV})$ & $\mathrm{mg} / \mathrm{kg}$ & $1.42 \mathrm{E}+04$ & $1.42 \mathrm{E}+04$ & $1.34 \mathrm{E}+04$ & $1.39 \mathrm{E}+04$ & $3.4 \%$ & $<1.1 \mathrm{E}+02$ & $<1.3 \mathrm{E}+02$ & - \\
\hline${ }^{233} \mathrm{U}$ (IM) & $\mathrm{mg} / \mathrm{kg}$ & $<5.1 \mathrm{E}+00$ & $<5.1 \mathrm{E}+00$ & $<5.0 \mathrm{E}+00$ & - & - & $<5.0 \mathrm{E}+00$ & $<6.0 \mathrm{E}+00$ & - \\
\hline${ }^{234} \mathrm{U}$ (IM) & $\mathrm{mg} / \mathrm{kg}$ & $<5.1 \mathrm{E}+00$ & $<5.1 \mathrm{E}+00$ & $<5.0 \mathrm{E}+00$ & - & - & $<5.0 \mathrm{E}+00$ & $<6.0 \mathrm{E}+00$ & - \\
\hline${ }^{235} \mathrm{U}$ (IM) & $\mathrm{mg} / \mathrm{kg}$ & $9.93 \mathrm{E}+01$ & $1.08 \mathrm{E}+02$ & $9.38 \mathrm{E}+01$ & $1.00 \mathrm{E}+02$ & $6.9 \%$ & $<5.0 \mathrm{E}+00$ & $<6.0 \mathrm{E}+00$ & - \\
\hline${ }^{236} \mathrm{U}$ (IM) & $\mathrm{mg} / \mathrm{kg}$ & $<5.1 \mathrm{E}+00$ & $<5.1 \mathrm{E}+00$ & $<5.0 \mathrm{E}+00$ & - & - & $<5.0 \mathrm{E}+00$ & $<6.0 \mathrm{E}+00$ & - \\
\hline${ }^{238} \mathrm{U}$ (IM) & $\mathrm{mg} / \mathrm{kg}$ & $1.27 \mathrm{E}+04$ & $1.31 \mathrm{E}+04$ & $1.23 \mathrm{E}+04$ & $1.27 \mathrm{E}+04$ & $3.2 \%$ & $<2.8 \mathrm{E}+01$ & $<3.3 \mathrm{E}+01$ & - \\
\hline
\end{tabular}

Divide $\mathrm{mg} / \mathrm{kg}$ values by $1 \mathrm{E}+04$ to convert to wt $\%$ dried solids basis 
WSRC-STI-2008-00227, REV. 0

Table 3-6. Composition of the Aqua Regia Dissolution of Total Dried Solids from the As-Received Tank 51H Sludge (Continued)

\begin{tabular}{|c|c|c|c|c|c|c|c|c|c|}
\hline Analyte (Method) & Units & $\begin{array}{c}\text { 1st } \\
\text { Replicate } \\
\end{array}$ & $\begin{array}{c}\text { 2nd } \\
\text { Replicate }\end{array}$ & $\begin{array}{c}\text { 3rd } \\
\text { Replicate } \\
\end{array}$ & Average & $\%$ RSD & Blank & $\begin{array}{c}\text { Average } \\
\text { Analyzed } \\
\text { Glass Std }\end{array}$ & $\begin{array}{c}\text { Glass Std } \\
\text { Composition }\end{array}$ \\
\hline${ }^{137}$ Cs (SG) & $\mathrm{mCi} / \mathrm{kg}$ & $1.17 \mathrm{E}+02$ & $1.10 \mathrm{E}+02$ & $1.10 \mathrm{E}+02$ & $1.12 \mathrm{E}+02$ & $3.4 \%$ & $<8.2 \mathrm{E}-02$ & $<9.5 \mathrm{E}-02$ & - \\
\hline${ }^{134}$ Cs (SG) & $\mathrm{mCi} / \mathrm{kg}$ & $<2.5 \mathrm{E}-01$ & $<2.5 \mathrm{E}-01$ & $<2.5 \mathrm{E}-01$ & - & - & $<7.2 \mathrm{E}-02$ & $<9.5 E-02$ & - \\
\hline${ }^{60}$ Co (SG) & $\mathrm{mCi} / \mathrm{kg}$ & 3.00E-01 & 2.86E-01 & 2.92E-01 & 2.93E-01 & $2.3 \%$ & $<3.0 \mathrm{E}-03$ & $<3.7 \mathrm{E}-03$ & - \\
\hline${ }^{106} \mathrm{Ru}(\mathrm{SG})$ & $\mathrm{mCi} / \mathrm{kg}$ & $<3.3 \mathrm{E}-01$ & $<3.3 \mathrm{E}-01$ & $<2.8 \mathrm{E}-01$ & - & - & $<1.7 \mathrm{E}-02$ & $<2.0 \mathrm{E}-02$ & - \\
\hline${ }^{125} \mathrm{Sb}(\mathrm{SG})$ & $\mathrm{mCi} / \mathrm{kg}$ & $<1.4 \mathrm{E}-01$ & $<1.4 \mathrm{E}-01$ & $<1.3 \mathrm{E}-01$ & - & - & $<7.8 \mathrm{E}-03$ & $<9.7 E-03$ & - \\
\hline${ }^{126} \mathrm{Sn}(\mathrm{SG})$ & $\mathrm{mCi} / \mathrm{kg}$ & $<2.3 \mathrm{E}-01$ & $<2.3 \mathrm{E}-01$ & $<2.3 \mathrm{E}-01$ & - & - & $<5.5 \mathrm{E}-03$ & $<6.4 \mathrm{E}-03$ & - \\
\hline${ }^{126} \mathrm{Sb}(\mathrm{SG})$ & $\mathrm{mCi} / \mathrm{kg}$ & $<4.5 \mathrm{E}-02$ & $<4.5 \mathrm{E}-02$ & $<4.5 \mathrm{E}-02$ & - & - & $<2.8 \mathrm{E}-03$ & $<3.1 \mathrm{E}-03$ & - \\
\hline${ }^{144} \mathrm{Ce}$ (SG) & $\mathrm{mCi} / \mathrm{kg}$ & $<5.2 \mathrm{E}-01$ & $<5.2 \mathrm{E}-01$ & $<5.2 \mathrm{E}-01$ & - & - & $<1.6 \mathrm{E}-02$ & $<1.9 \mathrm{E}-02$ & - \\
\hline${ }^{154} \mathrm{Eu}$ (SG) & $\mathrm{mCi} / \mathrm{kg}$ & $1.69 \mathrm{E}+01$ & $1.70 \mathrm{E}+01$ & $1.69 \mathrm{E}+01$ & $1.69 \mathrm{E}+01$ & $0.5 \%$ & $<3.8 \mathrm{E}-03$ & $<4.6 \mathrm{E}-03$ & - \\
\hline${ }^{155} \mathrm{Eu}$ (SG) & $\mathrm{mCi} / \mathrm{kg}$ & $1.63 \mathrm{E}+00$ & $<1.5 \mathrm{E}+00$ & $1.41 \mathrm{E}+00$ & $1.52 \mathrm{E}+00 *$ & $10 \%$ & $<6.4 \mathrm{E}-03$ & $<7.4 \mathrm{E}-03$ & - \\
\hline${ }^{241} \mathrm{Am}$ (SG) & $\mathrm{mCi} / \mathrm{kg}$ & $1.23 \mathrm{E}+01$ & $1.22 \mathrm{E}+01$ & $1.23 E+01$ & $1.23 \mathrm{E}+01$ & $0.2 \%$ & $<1.3 \mathrm{E}-02$ & $<1.6 \mathrm{E}-02$ & - \\
\hline${ }^{99}$ Tc (IM) & $\mathrm{mCi} / \mathrm{kg}$ & $<4.8 \mathrm{E}-01$ & $<4.8 \mathrm{E}-01$ & $<4.7 \mathrm{E}-01$ & - & - & $<4.7 \mathrm{E}-01$ & $<5.6 \mathrm{E}-01$ & - \\
\hline${ }^{237} \mathrm{~Np}$ (IM) & $\mathrm{mCi} / \mathrm{kg}$ & $<3.6 \mathrm{E}-03$ & $<3.6 \mathrm{E}-03$ & $<3.6 \mathrm{E}-03$ & - & - & $<3.5 \mathrm{E}-03$ & $<4.2 \mathrm{E}-03$ & - \\
\hline${ }^{239} \mathrm{Pu}$ (IM) & $\mathrm{mCi} / \mathrm{kg}$ & $1.19 \mathrm{E}+00$ & $1.28 \mathrm{E}+00$ & $1.22 \mathrm{E}+00$ & $1.23 \mathrm{E}+00$ & $3.7 \%$ & $<3.1 \mathrm{E}-01$ & $<3.7 \mathrm{E}-01$ & - \\
\hline${ }^{240} \mathrm{Pu}$ (IM) & $\mathrm{mCi} / \mathrm{kg}$ & $<1.2 \mathrm{E}+00$ & $<1.2 \mathrm{E}+00$ & $<1.1 \mathrm{E}+00$ & - & - & $<1.1 \mathrm{E}+00$ & $<1.4 \mathrm{E}+00$ & - \\
\hline${ }^{242} \mathrm{Pu}$ (IM) & $\mathrm{mCi} / \mathrm{kg}$ & $<2.0 \mathrm{E}-02$ & $<2.0 \mathrm{E}-02$ & $<2.0 \mathrm{E}-02$ & - & - & $<2.0 \mathrm{E}-02$ & $<2.4 \mathrm{E}-02$ & - \\
\hline${ }^{238} \mathrm{Pu}$ (SA) & $\mathrm{mCi} / \mathrm{kg}$ & $2.77 \mathrm{E}+02$ & $2.45 E+02$ & $2.34 \mathrm{E}+02$ & $2.52 \mathrm{E}+02$ & $8.8 \%$ & $<4.1 \mathrm{E}-01$ & $<6.8 \mathrm{E}-01$ & - \\
\hline${ }^{239 / 240} \mathrm{Pu}$ (SA) & $\mathrm{mCi} / \mathrm{kg}$ & $8.16 \mathrm{E}+00$ & $8.45 E+00$ & $7.17 \mathrm{E}+00$ & $7.93 \mathrm{E}+00$ & $8.4 \%$ & $<9.6 \mathrm{E}-01$ & 7.16E-01 & - \\
\hline${ }^{241} \mathrm{Pu}$ (SA) & $\mathrm{mCi} / \mathrm{kg}$ & $7.18 \mathrm{E}+01$ & $7.25 \mathrm{E}+01$ & $6.04 \mathrm{E}+01$ & $6.82 E+01$ & $10 \%$ & $<5.4 \mathrm{E}+00$ & $<5.2 \mathrm{E}+00$ & - \\
\hline
\end{tabular}

* Only two values averaged. 
WSRC-STI-2008-00227, REV. 0

Table 3-6. Composition of the Aqua Regia Dissolution of Total Dried Solids from the As-Received Tank 51H Sludge (Continued)

\begin{tabular}{|l|c|c|c|c|c|c|c|c|c|}
\hline Analyte (Method) & Units & $\begin{array}{c}\text { 1st } \\
\text { Replicate }\end{array}$ & $\begin{array}{c}\text { 2nd } \\
\text { Replicate }\end{array}$ & $\begin{array}{c}\text { 3rd } \\
\text { Replicate }\end{array}$ & Average & \%RSD & Blank & $\begin{array}{c}\text { Average } \\
\text { Analyzed } \\
\text { Glass Std }\end{array}$ & $\begin{array}{c}\text { Glass Std } \\
\text { Composition }\end{array}$ \\
\hline${ }^{90} \mathrm{Sr}$ (SL) & $\mathrm{mCi} / \mathrm{kg}$ & $5.24 \mathrm{E}+03$ & $5.63 \mathrm{E}+03$ & $6.40 \mathrm{E}+03$ & $5.76 \mathrm{E}+03$ & $10 \%$ & $<1.9 \mathrm{E}-02$ & $<8.2 \mathrm{E}-02$ & - \\
\hline Total Alpha (SL) & $\mathrm{mCi} / \mathrm{kg}$ & $3.02 \mathrm{E}+02$ & $3.17 \mathrm{E}+02$ & $2.94 \mathrm{E}+02$ & $3.04 \mathrm{E}+02$ & $3.8 \%$ & $<8.2 \mathrm{E}+00$ & $<1.1 \mathrm{E}+01$ & - \\
\hline Total Beta (SL) & $\mathrm{mCi} / \mathrm{kg}$ & $1.57 \mathrm{E}+04$ & $1.63 \mathrm{E}+04$ & $1.50 \mathrm{E}+04$ & $1.57 \mathrm{E}+04$ & $4.2 \%$ & $<2.3 \mathrm{E}+01$ & $<2.9 \mathrm{E}+01$ & - \\
\hline
\end{tabular}


WSRC-STI-2008-00227, REV. 0

Table 3-7. Composition of the Sodium Peroxide Fusion Dissolution of Total Dried Solids from the As-Received Tank 51H Sludge

\begin{tabular}{|c|c|c|c|c|c|c|c|c|c|}
\hline Analyte (Method) & Units & $\begin{array}{c}\text { 1st } \\
\text { Replicate } \\
\end{array}$ & $\begin{array}{c}\text { 2nd } \\
\text { Replicate }\end{array}$ & $\begin{array}{c}\text { 3rd } \\
\text { Replicate } \\
\end{array}$ & Average & \%RSD & Blank & $\begin{array}{c}\text { Average } \\
\text { Analyzed } \\
\text { Glass Std } \\
\end{array}$ & $\begin{array}{c}\text { Glass Std } \\
\text { Composition }\end{array}$ \\
\hline Ag (IE) & $\mathrm{mg} / \mathrm{kg}$ & $<9.9 \mathrm{E}+02$ & $<9.4 \mathrm{E}+02$ & $<9.4 \mathrm{E}+02$ & - & - & $<9.6 \mathrm{E}+02$ & $<1.1 \mathrm{E}+03$ & - \\
\hline $\mathrm{Al}$ (IE) & $\mathrm{mg} / \mathrm{kg}$ & $7.99 \mathrm{E}+04$ & $8.18 \mathrm{E}+04$ & $7.89 \mathrm{E}+04$ & $8.02 \mathrm{E}+04$ & $1.8 \%$ & $<1.3 \mathrm{E}+03$ & $2.54 \mathrm{E}+04$ & $2.50 \mathrm{E}+04$ \\
\hline B (IE) & $\mathrm{mg} / \mathrm{kg}$ & $<7.7 \mathrm{E}+02$ & $<7.3 \mathrm{E}+02$ & $<7.3 \mathrm{E}+02$ & - & - & $<7.5 \mathrm{E}+02$ & $2.52 \mathrm{E}+04$ & $2.69 \mathrm{E}+04$ \\
\hline $\mathrm{Ba}$ (IE) & $\mathrm{mg} / \mathrm{kg}$ & $3.56 \mathrm{E}+02$ & $4.03 E+02$ & $3.34 \mathrm{E}+02$ & $3.64 \mathrm{E}+02$ & $10 \%$ & $<6.3 \mathrm{E}+01$ & $8.15 E+02$ & $7.90 \mathrm{E}+02$ \\
\hline Be (IE) & $\mathrm{mg} / \mathrm{kg}$ & $<1.1 \mathrm{E}+01$ & $<1.1 \mathrm{E}+01$ & $<1.1 \mathrm{E}+01$ & - & - & $<1.1 \mathrm{E}+01$ & $<1.3 \mathrm{E}+01$ & - \\
\hline $\mathrm{Ca}$ (IE) & $\mathrm{mg} / \mathrm{kg}$ & $6.85 \mathrm{E}+03$ & $8.00 \mathrm{E}+03$ & $6.36 \mathrm{E}+03$ & $7.07 \mathrm{E}+03$ & $12 \%$ & $1.45 E+03$ & $1.15 E+04$ & $1.02 \mathrm{E}+04$ \\
\hline Cd (IE) & $\mathrm{mg} / \mathrm{kg}$ & $2.08 \mathrm{E}+02$ & $2.24 \mathrm{E}+02$ & $1.91 \mathrm{E}+02$ & $2.07 \mathrm{E}+02$ & $7.9 \%$ & $<8.2 \mathrm{E}+01$ & $<9.6 \mathrm{E}+01$ & - \\
\hline Ce (IE) & $\mathrm{mg} / \mathrm{kg}$ & $<3.3 \mathrm{E}+03$ & $<3.1 \mathrm{E}+03$ & $<3.1 \mathrm{E}+03$ & - & - & $<3.2 \mathrm{E}+03$ & $<3.7 \mathrm{E}+03$ & - \\
\hline Cr (IE) & $\mathrm{mg} / \mathrm{kg}$ & $3.45 \mathrm{E}+02$ & $3.71 E+02$ & $3.44 \mathrm{E}+02$ & $3.53 \mathrm{E}+02$ & $4.4 \%$ & $<1.0 \mathrm{E}+02$ & $8.19 \mathrm{E}+02$ & $6.40 \mathrm{E}+02$ \\
\hline $\mathrm{Cu}$ (IE) & $\mathrm{mg} / \mathrm{kg}$ & $1.95 \mathrm{E}+02$ & $2.17 \mathrm{E}+02$ & $1.97 \mathrm{E}+02$ & $2.03 \mathrm{E}+02$ & $5.9 \%$ & $<1.7 \mathrm{E}+02$ & $<1.9 \mathrm{E}+02$ & $3.00 \mathrm{E}+01$ \\
\hline Fe (IE) & $\mathrm{mg} / \mathrm{kg}$ & $5.67 \mathrm{E}+04$ & $6.52 E+04$ & $5.17 \mathrm{E}+04$ & $5.79 \mathrm{E}+04$ & $12 \%$ & $2.61 \mathrm{E}+02$ & $9.94 \mathrm{E}+04$ & $9.79 \mathrm{E}+04$ \\
\hline Gd (IE) & $\mathrm{mg} / \mathrm{kg}$ & $<3.6 \mathrm{E}+02$ & $<3.4 \mathrm{E}+02$ & $<3.4 \mathrm{E}+02$ & - & - & $<3.5 \mathrm{E}+02$ & $<4.0 \mathrm{E}+02$ & - \\
\hline K (IE) & $\mathrm{mg} / \mathrm{kg}$ & $<4.9 \mathrm{E}+03$ & $<4.7 \mathrm{E}+03$ & $<4.7 \mathrm{E}+03$ & - & - & $<4.8 \mathrm{E}+03$ & $2.28 \mathrm{E}+04$ & $2.26 \mathrm{E}+04$ \\
\hline La (IE) & $\mathrm{mg} / \mathrm{kg}$ & $<4.4 \mathrm{E}+02$ & $<4.2 \mathrm{E}+02$ & $<4.2 \mathrm{E}+02$ & - & - & $<4.3 \mathrm{E}+02$ & $<4.9 \mathrm{E}+02$ & - \\
\hline Li (IE) & $\mathrm{mg} / \mathrm{kg}$ & $<3.0 \mathrm{E}+02$ & $<2.9 \mathrm{E}+02$ & $<2.9 \mathrm{E}+02$ & - & - & $<2.9 \mathrm{E}+02$ & $1.48 \mathrm{E}+04$ & $1.49 \mathrm{E}+04$ \\
\hline $\mathrm{Mg}$ (IE) & $\mathrm{mg} / \mathrm{kg}$ & $2.24 \mathrm{E}+03$ & $2.57 E+03$ & $1.99 \mathrm{E}+03$ & $2.27 \mathrm{E}+03$ & $13 \%$ & $5.02 \mathrm{E}+01$ & $5.14 \mathrm{E}+03$ & $5.20 \mathrm{E}+03$ \\
\hline Mn (IE) & $\mathrm{mg} / \mathrm{kg}$ & $1.31 \mathrm{E}+04$ & $1.49 E+04$ & $1.18 \mathrm{E}+04$ & $1.33 E+04$ & $12 \%$ & $<9.6 \mathrm{E}+01$ & $1.39 \mathrm{E}+04$ & $1.46 \mathrm{E}+04$ \\
\hline Mo (IE) & $\mathrm{mg} / \mathrm{kg}$ & $<3.3 \mathrm{E}+02$ & $<3.1 \mathrm{E}+02$ & $<3.1 \mathrm{E}+02$ & - & - & $<3.2 \mathrm{E}+02$ & $<3.7 \mathrm{E}+02$ & - \\
\hline $\mathrm{Ni}$ (IE) & $\mathrm{mg} / \mathrm{kg}$ & $4.40 \mathrm{E}+03$ & $4.96 \mathrm{E}+03$ & $3.95 \mathrm{E}+03$ & $4.44 \mathrm{E}+03$ & $11 \%$ & $<5.1 \mathrm{E}+02$ & $7.57 \mathrm{E}+03$ & $8.27 \mathrm{E}+03$ \\
\hline
\end{tabular}

Divide $\mathrm{mg} / \mathrm{kg}$ values by $1 \mathrm{E}+04$ to convert to wt \% dried solids basis 
WSRC-STI-2008-00227, REV. 0

Table 3-7. Composition of the Sodium Peroxide Fusion Dissolution of Total Dried Solids from the As-Received Tank 51H Sludge (Continued)

\begin{tabular}{|c|c|c|c|c|c|c|c|c|c|}
\hline Analyte (Method) & Units & $\begin{array}{c}\text { 1st } \\
\text { Replicate }\end{array}$ & $\begin{array}{c}\text { 2nd } \\
\text { Replicate }\end{array}$ & $\begin{array}{c}\text { 3rd } \\
\text { Replicate }\end{array}$ & Average & \%RSD & Blank & $\begin{array}{l}\text { Average } \\
\text { Analyzed } \\
\text { Glass Std }\end{array}$ & $\begin{array}{c}\text { Glass Std } \\
\text { Composition }\end{array}$ \\
\hline $\mathrm{P}$ (IE) & $\mathrm{mg} / \mathrm{kg}$ & $1.13 \mathrm{E}+03$ & $1.20 \mathrm{E}+03$ & $8.70 \mathrm{E}+02$ & $1.07 \mathrm{E}+03$ & $16 \%$ & $<7.5 \mathrm{E}+02$ & $9.41 \mathrm{E}+02$ & $1.10 \mathrm{E}+03$ \\
\hline $\mathrm{Pb}$ (IE) & $\mathrm{mg} / \mathrm{kg}$ & $<8.5 \mathrm{E}+02$ & $<8.1 \mathrm{E}+02$ & $<8.1 \mathrm{E}+02$ & - & - & $<8.3 \mathrm{E}+02$ & $<9.6 \mathrm{E}+02$ & - \\
\hline S (IE) & $\mathrm{mg} / \mathrm{kg}$ & $<1.2 \mathrm{E}+04$ & $<1.2 \mathrm{E}+04$ & $<1.2 \mathrm{E}+04$ & - & - & $<1.2 \mathrm{E}+04$ & $<1.4 \mathrm{E}+04$ & - \\
\hline Sb (IE) & $\mathrm{mg} / \mathrm{kg}$ & $<1.6 \mathrm{E}+03$ & $<1.5 \mathrm{E}+03$ & $<1.6 \mathrm{E}+03$ & - & - & $<1.6 \mathrm{E}+03$ & $<1.8 \mathrm{E}+03$ & - \\
\hline Si (IE) & $\mathrm{mg} / \mathrm{kg}$ & $3.06 \mathrm{E}+03$ & $3.28 \mathrm{E}+03$ & $3.28 \mathrm{E}+03$ & $3.20 \mathrm{E}+03$ & $4.0 \%$ & $<2.9 \mathrm{E}+03$ & $2.24 \mathrm{E}+05$ & $2.24 \mathrm{E}+05$ \\
\hline Sn (IE) & $\mathrm{mg} / \mathrm{kg}$ & $<4.8 \mathrm{E}+03$ & $<4.6 \mathrm{E}+03$ & $<4.6 \mathrm{E}+03$ & - & - & $<4.7 \mathrm{E}+03$ & $<5.5 \mathrm{E}+03$ & - \\
\hline Sr (IE) & $\mathrm{mg} / \mathrm{kg}$ & $<4.3 \mathrm{E}+02$ & $<4.1 \mathrm{E}+02$ & $<4.1 \mathrm{E}+02$ & - & - & $<4.2 \mathrm{E}+02$ & $<4.9 \mathrm{E}+02$ & $3.00 \mathrm{E}+01$ \\
\hline Ti (IE) & $\mathrm{mg} / \mathrm{kg}$ & $<9.9 \mathrm{E}+01$ & $<9.4 \mathrm{E}+01$ & $<9.4 \mathrm{E}+01$ & - & - & $<9.6 \mathrm{E}+01$ & $6.44 \mathrm{E}+03$ & $6.90 \mathrm{E}+03$ \\
\hline U (IE) & $\mathrm{mg} / \mathrm{kg}$ & $<2.0 \mathrm{E}+04$ & $<1.9 \mathrm{E}+04$ & $<1.9 \mathrm{E}+04$ & - & - & $<1.9 \mathrm{E}+04$ & $<2.2 \mathrm{E}+04$ & - \\
\hline $\mathrm{V}$ (IE) & $\mathrm{mg} / \mathrm{kg}$ & $<1.9 \mathrm{E}+02$ & $<1.8 \mathrm{E}+02$ & $<1.8 \mathrm{E}+02$ & - & - & $<1.8 \mathrm{E}+02$ & $<2.1 \mathrm{E}+02$ & - \\
\hline $\mathrm{Zn}$ (IE) & $\mathrm{mg} / \mathrm{kg}$ & $2.52 \mathrm{E}+02$ & $3.47 \mathrm{E}+02$ & $2.91 \mathrm{E}+02$ & $2.97 \mathrm{E}+02$ & $16 \%$ & $1.41 \mathrm{E}+02$ & $2.27 \mathrm{E}+02$ & $1.60 \mathrm{E}+02$ \\
\hline${ }^{232} \mathrm{U}(\mathrm{SA})$ & $\mathrm{mg} / \mathrm{kg}$ & $<1.6 \mathrm{E}-04$ & $<1.0 \mathrm{E}-04$ & $<8.8 \mathrm{E}-05$ & - & - & $<1.1 \mathrm{E}-05$ & $<3.5 \mathrm{E}-05$ & - \\
\hline${ }^{233} \mathrm{U}$ (IM) & $\mathrm{mg} / \mathrm{kg}$ & $<5.1 \mathrm{E}+00$ & $<4.9 \mathrm{E}+00$ & $<4.9 \mathrm{E}+00$ & - & - & $<5.0 \mathrm{E}+00$ & $<5.8 \mathrm{E}+00$ & - \\
\hline${ }^{234} \mathrm{U}$ (IM) & $\mathrm{mg} / \mathrm{kg}$ & $<5.1 \mathrm{E}+00$ & $<4.9 \mathrm{E}+00$ & $<4.9 \mathrm{E}+00$ & - & - & $<5.0 \mathrm{E}+00$ & $<5.8 \mathrm{E}+00$ & - \\
\hline${ }^{235} \mathrm{U}$ (IM) & $\mathrm{mg} / \mathrm{kg}$ & $9.42 \mathrm{E}+01$ & $1.12 \mathrm{E}+02$ & $8.39 \mathrm{E}+01$ & $9.68 \mathrm{E}+01$ & $15 \%$ & $<5.0 \mathrm{E}+00$ & $<5.8 \mathrm{E}+00$ & - \\
\hline${ }^{236} \mathrm{U}$ (IM) & $\mathrm{mg} / \mathrm{kg}$ & $<5.1 \mathrm{E}+00$ & $<4.9 \mathrm{E}+00$ & $<4.9 \mathrm{E}+00$ & - & - & $<5.0 \mathrm{E}+00$ & $<5.8 \mathrm{E}+00$ & - \\
\hline${ }^{238} \mathrm{U}$ (IM) & $\mathrm{mg} / \mathrm{kg}$ & $1.23 \mathrm{E}+04$ & $1.41 \mathrm{E}+04$ & $1.10 \mathrm{E}+04$ & $1.25 \mathrm{E}+04$ & $12 \%$ & $<1.3 \mathrm{E}+01$ & $<1.5 \mathrm{E}+01$ & - \\
\hline
\end{tabular}

Divide $\mathrm{mg} / \mathrm{kg}$ values by $1 \mathrm{E}+04$ to convert to wt \% dried solids basis 
WSRC-STI-2008-00227, REV. 0

Table 3-7. Composition of the Sodium Peroxide Fusion Dissolution of Total Dried Solids from the As-Received Tank 51H Sludge (Continued)

\begin{tabular}{|c|c|c|c|c|c|c|c|c|c|}
\hline Analyte (Method) & Units & $\begin{array}{c}\text { 1st } \\
\text { Replicate } \\
\end{array}$ & $\begin{array}{c}\text { 2nd } \\
\text { Replicate }\end{array}$ & $\begin{array}{c}\text { 3rd } \\
\text { Replicate }\end{array}$ & Average & \%RSD & Blank & $\begin{array}{l}\text { Average } \\
\text { Analyzed } \\
\text { Glass Std }\end{array}$ & $\begin{array}{c}\text { Glass Std } \\
\text { Composition }\end{array}$ \\
\hline${ }^{137} \mathrm{Cs}$ (SG) & $\mathrm{mCi} / \mathrm{kg}$ & $1.15 \mathrm{E}+02$ & $1.17 \mathrm{E}+02$ & $1.13 \mathrm{E}+02$ & $1.15 \mathrm{E}+02$ & $1.5 \%$ & $1.16 \mathrm{E}-01$ & $1.66 \mathrm{E}-01$ & - \\
\hline${ }^{134}$ Cs (SG) & $\mathrm{mCi} / \mathrm{kg}$ & $<2.5 \mathrm{E}-01$ & $<2.7 \mathrm{E}-01$ & $<2.4 \mathrm{E}-01$ & - & - & $<6.6 \mathrm{E}-02$ & $<9.5 \mathrm{E}-02$ & - \\
\hline${ }^{60}$ Co (SG) & $\mathrm{mCi} / \mathrm{kg}$ & $3.04 \mathrm{E}-01$ & $3.42 \mathrm{E}-01$ & $2.76 \mathrm{E}-01$ & $3.08 \mathrm{E}-01$ & $11 \%$ & $<2.4 \mathrm{E}-03$ & $<2.6 \mathrm{E}-03$ & - \\
\hline${ }^{106} \mathrm{Ru}$ (SG) & $\mathrm{mCi} / \mathrm{kg}$ & $<2.3 \mathrm{E}-01$ & $<2.5 \mathrm{E}-01$ & $<2.3 \mathrm{E}-01$ & - & - & $<1.3 \mathrm{E}-02$ & $<1.8 \mathrm{E}-02$ & - \\
\hline${ }^{125} \mathrm{Sb}(\mathrm{SG})$ & $\mathrm{mCi} / \mathrm{kg}$ & $7.28 \mathrm{E}-02$ & $<7.0 \mathrm{E}-02$ & $<9.5 \mathrm{E}-02$ & - & - & $<5.4 \mathrm{E}-03$ & $<6.2 \mathrm{E}-03$ & - \\
\hline${ }^{126} \mathrm{Sn}$ (SG) & $\mathrm{mCi} / \mathrm{kg}$ & $<2.0 \mathrm{E}-01$ & $<2.0 \mathrm{E}-01$ & $<1.8 \mathrm{E}-01$ & - & - & $<4.1 \mathrm{E}-03$ & $<4.6 \mathrm{E}-03$ & - \\
\hline${ }^{126} \mathrm{Sb}(\mathrm{SG})$ & $\mathrm{mCi} / \mathrm{kg}$ & $<3.5 \mathrm{E}-02$ & $<3.7 \mathrm{E}-02$ & $<3.2 \mathrm{E}-02$ & - & - & $<1.9 \mathrm{E}-03$ & $<2.2 \mathrm{E}-03$ & - \\
\hline${ }^{144} \mathrm{Ce}$ (SG) & $\mathrm{mCi} / \mathrm{kg}$ & $<4.1 \mathrm{E}-01$ & $<4.3 \mathrm{E}-01$ & $<3.8 \mathrm{E}-01$ & - & - & $<1.0 \mathrm{E}-02$ & $<1.3 \mathrm{E}-02$ & - \\
\hline${ }^{154} \mathrm{Eu}(\mathrm{SG})$ & $\mathrm{mCi} / \mathrm{kg}$ & $1.72 \mathrm{E}+01$ & $2.00 \mathrm{E}+01$ & $1.54 \mathrm{E}+01$ & $1.75 \mathrm{E}+01$ & $13 \%$ & $<2.8 \mathrm{E}-03$ & $<3.6 \mathrm{E}-03$ & - \\
\hline${ }^{155} \mathrm{Eu}$ (SG) & $\mathrm{mCi} / \mathrm{kg}$ & $1.64 \mathrm{E}+00$ & $<2.1 \mathrm{E}+00$ & $1.23 \mathrm{E}+00$ & $1.44 \mathrm{E}+00 *$ & $20 \%$ & $<4.9 \mathrm{E}-03$ & $<5.5 \mathrm{E}-03$ & - \\
\hline${ }^{241} \mathrm{Am}$ (SG) & $\mathrm{mCi} / \mathrm{kg}$ & $1.15 \mathrm{E}+01$ & $1.38 \mathrm{E}+01$ & $1.03 \mathrm{E}+01$ & $1.19 \mathrm{E}+01$ & $15 \%$ & 1.91E-02 & $1.67 \mathrm{E}-01$ & - \\
\hline${ }^{99}$ Tc (IM) & $\mathrm{mCi} / \mathrm{kg}$ & $<3.1 \mathrm{E}-01$ & $<2.9 \mathrm{E}-01$ & $<2.9 \mathrm{E}-01$ & - & - & $<3.0 \mathrm{E}-01$ & $<3.5 \mathrm{E}-01$ & - \\
\hline${ }^{237} \mathrm{~Np}$ (IM) & $\mathrm{mCi} / \mathrm{kg}$ & $<3.6 \mathrm{E}-03$ & 3.61E-03 & $<3.5 \mathrm{E}-03$ & - & - & $<3.5 \mathrm{E}-03$ & $<4.1 \mathrm{E}-03$ & - \\
\hline${ }^{239} \mathrm{Pu}$ (IM) & $\mathrm{mCi} / \mathrm{kg}$ & $1.64 \mathrm{E}+00$ & $2.10 \mathrm{E}+00$ & $1.54 \mathrm{E}+00$ & $1.76 \mathrm{E}+00$ & $17 \%$ & $<3.1 \mathrm{E}-01$ & $<3.6 \mathrm{E}-01$ & - \\
\hline${ }^{240} \mathrm{Pu}$ (IM) & $\mathrm{mCi} / \mathrm{kg}$ & $<1.2 \mathrm{E}+00$ & $<1.1 \mathrm{E}+00$ & $<1.1 \mathrm{E}+00$ & - & - & $<1.1 \mathrm{E}+00$ & $<1.3 \mathrm{E}+00$ & - \\
\hline${ }^{242} \mathrm{Pu}$ (IM) & $\mathrm{mCi} / \mathrm{kg}$ & $<2.0 \mathrm{E}-02$ & $<1.9 \mathrm{E}-02$ & $<1.9 \mathrm{E}-02$ & - & - & $<2.0 \mathrm{E}-02$ & $<2.3 \mathrm{E}-02$ & - \\
\hline${ }^{238} \mathrm{Pu}$ (SA) & $\mathrm{mCi} / \mathrm{kg}$ & $1.94 \mathrm{E}+02$ & $2.07 \mathrm{E}+02$ & $1.80 \mathrm{E}+02$ & $1.93 \mathrm{E}+02$ & $7.0 \%$ & $<6.1 \mathrm{E}-03$ & $<3.1 \mathrm{E}-02$ & - \\
\hline${ }^{239 / 240} \mathrm{Pu}$ (SA) & $\mathrm{mCi} / \mathrm{kg}$ & $5.47 \mathrm{E}+00$ & $5.63 \mathrm{E}+00$ & $4.61 \mathrm{E}+00$ & $5.24 \mathrm{E}+00$ & $10 \%$ & $2.27 \mathrm{E}-02$ & $<8.6 \mathrm{E}-03$ & - \\
\hline${ }^{241} \mathrm{Pu}(\mathrm{SA})$ & $\mathrm{mCi} / \mathrm{kg}$ & $6.16 \mathrm{E}+01$ & $6.29 \mathrm{E}+01$ & $4.70 \mathrm{E}+01$ & $5.72 \mathrm{E}+01$ & $15 \%$ & $5.41 \mathrm{E}-02$ & $<3.5 \mathrm{E}-02$ & - \\
\hline
\end{tabular}

* Only two values averaged. 
WSRC-STI-2008-00227, REV. 0

Table 3-7. Composition of the Sodium Peroxide Fusion Dissolution of Total Dried Solids from the As-Received Tank 51H Sludge (Continued)

\begin{tabular}{|c|c|c|c|c|c|c|c|c|c|}
\hline Analyte (Method) & Units & $\begin{array}{c}\text { 1st } \\
\text { Replicate } \\
\end{array}$ & $\begin{array}{c}\text { 2nd } \\
\text { Replicate } \\
\end{array}$ & $\begin{array}{c}\text { 3rd } \\
\text { Replicate }\end{array}$ & Average & \%RSD & Blank & $\begin{array}{l}\text { Average } \\
\text { Analyzed } \\
\text { Glass Std }\end{array}$ & $\begin{array}{c}\text { Glass Std } \\
\text { Composition }\end{array}$ \\
\hline${ }^{90} \mathrm{Sr}(\mathrm{SL})$ & $\mathrm{mCi} / \mathrm{kg}$ & $5.01 \mathrm{E}+03$ & $5.67 \mathrm{E}+03$ & $4.70 \mathrm{E}+03$ & $5.13 \mathrm{E}+03$ & $9.7 \%$ & 9.55E-01 & $1.25 \mathrm{E}+00$ & - \\
\hline${ }^{79}$ Se (SL)* & $\mathrm{mCi} / \mathrm{kg}$ & 2.22E-02 & 2.89E-02 & 3.80E-02 & 2.97E-02 & $27 \%$ & - & - & - \\
\hline${ }^{129} \mathrm{I}(\mathrm{SL}) *$ & $\mathrm{mCi} / \mathrm{kg}$ & 8.60E-04 & 8.43E-04 & $7.06 \mathrm{E}-04$ & 8.03E-04 & $11 \%$ & - & - & - \\
\hline Total Alpha (SL) & $\mathrm{mCi} / \mathrm{kg}$ & $2.86 \mathrm{E}+02$ & $3.18 \mathrm{E}+02$ & $2.77 \mathrm{E}+02$ & $2.94 \mathrm{E}+02$ & $7.4 \%$ & $<9.7 \mathrm{E}+00$ & $<1.3 \mathrm{E}+01$ & - \\
\hline Total Beta (SL) & $\mathrm{mCi} / \mathrm{kg}$ & $1.39 E+04$ & $1.61 \mathrm{E}+04$ & $1.17 \mathrm{E}+04$ & $1.39 E+04$ & $16 \%$ & $<2.2 \mathrm{E}+01$ & $<2.6 \mathrm{E}+01$ & - \\
\hline
\end{tabular}

* The Se-79 and I-129 required special sample preparations and are reported with the sodium peroxide fusion results only for convenience. 


\subsection{DISCUSSION OF RESULTS}

\subsection{AS-RECEIVED TANK 11H AND TANK 51H SUPERNATE CHARACTERIZATION}

The characterization results for the as-received Tank $11 \mathrm{H}$ and Tank $51 \mathrm{H}$ supernate appear quite good with respect to the precision of the sample replicates and minimal contamination present in the blank. Table 4-1 summarizes the composition of the key components of both samples using the data from Tables 3-4 and 3-5. As expected both supernate samples show similar compositions for the major components.

Table 4-1. Comparison of the Tank $11 \mathrm{H}$ and Tank 51H Supernate

\begin{tabular}{|c|c|c|c|c|c|}
\hline $\begin{array}{l}\text { Analyte } \\
\text { (Method) }\end{array}$ & Units & $\begin{array}{c}\text { Tank 11H } \\
\text { Supernate } \\
\text { (Average) }\end{array}$ & $\begin{array}{c}\text { Tank 11H } \\
\text { Supernate } \\
\text { (\%RSD) }\end{array}$ & $\begin{array}{l}\text { Tank 51H } \\
\text { Supernate } \\
\text { (Average) }\end{array}$ & $\begin{array}{c}\text { Tank 51H } \\
\text { Supernate } \\
\text { (\%RSD) }\end{array}$ \\
\hline $\mathrm{NO}_{3}^{-}$(IC) & M & $1.75 \mathrm{E}-01$ & $0.5 \%$ & $1.91 \mathrm{E}-01$ & $5.0 \%$ \\
\hline $\mathrm{NO}_{2}^{-}$(IC) & M & 3.30E-01 & $1.0 \%$ & 3.63E-01 & $3.9 \%$ \\
\hline $\mathrm{SO}_{4}{ }^{2-}(\mathrm{IC})$ & M & $1.74 \mathrm{E}-02$ & $0.8 \%$ & 1.79E-02 & $1.7 \%$ \\
\hline $\mathrm{Cl}^{-}$(IC) & $\mathrm{M}$ & $2.97 \mathrm{E}-02$ & $3.8 \%$ & $2.91 \mathrm{E}-02$ & $0.8 \%$ \\
\hline $\mathrm{OH}_{\text {free }}^{-}(\mathrm{TH})$ & $\mathrm{M}$ & $3.10 \mathrm{E}+00$ & $1.1 \%$ & $3.11 \mathrm{E}+00$ & $1.3 \%$ \\
\hline $\mathrm{CO}_{3}{ }^{2-}$ (TIC) & $\mathrm{M}$ & $9.81 \mathrm{E}-02$ & $1.3 \%$ & 1.27E-01 & $23 \%$ \\
\hline Al (IE) & $\mathrm{mg} / \mathrm{L}$ & $1.06 \mathrm{E}+04$ & $0.2 \%$ & $1.12 \mathrm{E}+04$ & $0.4 \%$ \\
\hline $\mathrm{Cr}$ (IE) & $\mathrm{mg} / \mathrm{L}$ & $2.77 \mathrm{E}+01$ & $0.6 \%$ & $2.60 \mathrm{E}+01$ & $0.1 \%$ \\
\hline Fe (IE) & $\mathrm{mg} / \mathrm{L}$ & $9.87 \mathrm{E}+00$ & $0.9 \%$ & $2.52 \mathrm{E}+00^{*}$ & - \\
\hline K (IE) & $\mathrm{mg} / \mathrm{L}$ & $1.29 \mathrm{E}+02$ & $4.0 \%$ & $1.60 \mathrm{E}+02$ & $3.7 \%$ \\
\hline $\mathrm{Na}$ (IE) & $\mathrm{mg} / \mathrm{L}$ & $9.58 \mathrm{E}+04$ & $0.4 \%$ & $9.32 E+04$ & $0.5 \%$ \\
\hline S (IE) & $\mathrm{mg} / \mathrm{L}$ & $5.94 \mathrm{E}+02$ & $1.1 \%$ & $5.92 \mathrm{E}+02$ & $1.8 \%$ \\
\hline $\mathrm{Hg}(\mathrm{CV})$ & $\mathrm{mg} / \mathrm{L}$ & $1.80 \mathrm{E}+02$ & $0.2 \%$ & $1.26 \mathrm{E}+02$ & $0.8 \%$ \\
\hline${ }^{238} \mathrm{U}$ (IM) & $\mathrm{mg} / \mathrm{L}$ & $4.74 \mathrm{E}+00$ & $11 \%$ & $1.79 \mathrm{E}+00$ & $2.3 \%$ \\
\hline${ }^{137} \mathrm{Cs}$ (SG) & $\mu \mathrm{Ci} / \mathrm{mL}$ & $1.49 \mathrm{E}+01$ & $2.3 \%$ & $1.50 \mathrm{E}+01$ & $0.6 \%$ \\
\hline${ }^{90} \mathrm{Sr}$ (SL) & $\mu \mathrm{Ci} / \mathrm{mL}$ & $6.56 \mathrm{E}-01$ & $2.8 \%$ & 3.84E-01 & $2.1 \%$ \\
\hline${ }^{238} \mathrm{Pu}(\mathrm{SA})$ & $\mu \mathrm{Ci} / \mathrm{mL}$ & $4.57 \mathrm{E}-02$ & $13 \%$ & $1.08 \mathrm{E}-01$ & $8.3 \%$ \\
\hline${ }^{239 / 240} \mathrm{Pu}(\mathrm{SA})$ & $\mu \mathrm{Ci} / \mathrm{mL}$ & $1.88 \mathrm{E}-03$ & $14 \%$ & 5.13E-03 & $7.2 \%$ \\
\hline${ }^{99}$ Tc (IM) & $\mu \mathrm{Ci} / \mathrm{mL}$ & $6.45 \mathrm{E}-03$ & $12 \%$ & 7.32E-03 & $12 \%$ \\
\hline
\end{tabular}

The As-Received Tank 11 Supernate was not filtered prior to submitting samples for analysis at the request of the customer. The Tank $51 \mathrm{H}$ supernate was filtered before analysis.

* Only a single replicate was above the detection limit. 


\subsection{AS-RECEIVED TANK 51H SLUDGE SAMPLE CHARACTERIZATION}

Table 4-2 summarizes the composition of the key components of the post aluminum dissolution sludge using the data from aqua regia and peroxide fusion dissolution of the total dried solids in Tables 3-6 and 3-7. The characterization results for the total dried solids of the Tank $51 \mathrm{H}$ sludge slurry sample appear good also. The sample replicates show good precision with minimal contamination present in the blanks and glass standards.

Table 4-2. Summary of the Post Aluminum Dissolution Tank 51H Sludge Composition

\begin{tabular}{|l|c|c|}
\hline Analyte & Average & \%RSD \\
\hline $\mathrm{Na} \mathrm{mg} / \mathrm{kg}$ & $3.03 \mathrm{E}+05$ & $0.4 \%$ \\
\hline Al mg/kg & $8.03 \mathrm{E}+04$ & $1.6 \%$ \\
\hline Fe mg/kg & $5.85 \mathrm{E}+04$ & $7.7 \%$ \\
\hline $\mathrm{Hg} \mathrm{mg} / \mathrm{kg}$ & $1.39 \mathrm{E}+04$ & $3.4 \%$ \\
\hline $\mathrm{Mn} \mathrm{mg} / \mathrm{kg}$ & $1.35 \mathrm{E}+04$ & $7.7 \%$ \\
\hline $\mathrm{U} \mathrm{mg} / \mathrm{kg}$ & $1.27 \mathrm{E}+04$ & $8.0 \%$ \\
\hline $\mathrm{Ca} \mathrm{mg} / \mathrm{kg}$ & $6.87 \mathrm{E}+03$ & $8.4 \%$ \\
\hline $\mathrm{Ni} \mathrm{mg} / \mathrm{kg}$ & $4.55 \mathrm{E}+03$ & $7.7 \%$ \\
\hline $\mathrm{Si} \mathrm{mg} / \mathrm{kg}$ & $3.20 \mathrm{E}+03$ & $4.0 \%$ \\
\hline $\mathrm{Mg} \mathrm{mg} / \mathrm{kg}$ & $2.37 \mathrm{E}+03$ & $9.2 \%$ \\
\hline${ }^{235} \mathrm{U} \mathrm{mg} / \mathrm{kg}$ & $9.85 \mathrm{E}+01$ & $10 \%$ \\
\hline${ }^{238} \mathrm{U} \mathrm{mg} / \mathrm{kg}$ & $1.26 \mathrm{E}+04$ & $8.0 \%$ \\
\hline${ }^{90} \mathrm{Sr} \mathrm{mCi} / \mathrm{kg}$ & $5.44 \mathrm{E}+03$ & $11 \%$ \\
\hline${ }^{99} \mathrm{Tc} \mathrm{mCi} / \mathrm{kg}$ & $<4.8 \mathrm{E}-01$ & - \\
\hline${ }^{137} \mathrm{Cs} \mathrm{mCi} / \mathrm{kg}$ & $1.14 \mathrm{E}+02$ & $2.7 \%$ \\
\hline${ }^{237} \mathrm{~Np} \mathrm{mCi} / \mathrm{kg}$ & $<3.6 \mathrm{E}-03$ & - \\
\hline${ }^{238} \mathrm{Pu} \mathrm{mCi} / \mathrm{kg}$ & $2.23 \mathrm{E}+02$ & $16 \%$ \\
\hline${ }^{239 / 240} \mathrm{Pu} \mathrm{mCi} / \mathrm{kg}$ & $6.58 \mathrm{E}+00$ & $24 \%$ \\
\hline${ }^{241} \mathrm{Pu} \mathrm{mCi} / \mathrm{kg}$ & $6.27 \mathrm{E}+01$ & $15 \%$ \\
\hline
\end{tabular}

Divide $\mathrm{mg} / \mathrm{kg}$ values by $1 \mathrm{E}+04$ to convert to wt \% dried solids basis

All results are the average of six replicates except $\mathrm{Na}, \mathrm{Hg}$ (only aqua regia data) and $\mathrm{Si}$ (only sodium peroxide fusion data). The \%RSD was calculated based on the same number of replicates as the average. 


\subsection{REFERENCES}

1. T. M. Punch, "Perform Sample Analysis for Samples Taken During Low Temperature Aluminum Dissolution (LTAD)” PIT-TTR-2207-00006, Rev. 0, October 24, 2007.

2. M. S. Hay, D. J. McCabe, “Task Technical and Quality Assurance Plan for Analysis of Low Temperature Aluminum Dissolution Samples from Tanks 11 and 51” WSRCRP-2007-01313, Rev. 0, December 13, 2007.

3. C. J. Coleman, R. A. Dewberry, M. F. Bryant, J. J. Gemmill, "SRL's Performance in Round Robin \#6 - Analysis of Simulated Defense Waste Glass (U)”, WSRC-TR-91187, Rev. 0, May 31, 1991.

4. M. S. Hay, D. J. McCabe, "Preliminary Characterization Data for the Tank $51 H$ and Tank 11H Post Aluminum Dissolution Process Samples”, SRNL-CST-2008-00015, Rev. 0, March 17, 2008.

5. M. S. Hay, T. B. Edwards, "Statistical Analysis of ESP Verification Test Samples”, WSRC-RP-94-1224, November 4, 1994.

6. M. S. Hay, J. M. Pareizs, C. J. Bannochie, M. E. Stone, D. R. Click, D. J. McCabe, "Characterization and Aluminum Dissolution Demonstration with a 3 Liter Tank 51H Sample”, WSRC-STI-2007-00697, February 2008. 